\title{
X-ray Spectral and Timing evolution of MAXI J1727-203 with NICER
}

\author{
K. Alabarta ${ }^{1,2 \star}$, D. Altamirano ${ }^{1}$, M. Méndez ${ }^{2}$, V. A. Cúneo ${ }^{3,4}$, L. Zhang ${ }^{1}$,

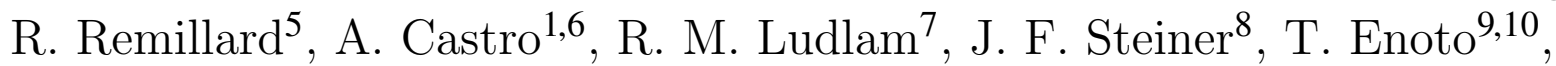
J. Homan ${ }^{11,12}$, Z. Arzoumanian ${ }^{14}$, P. Bult ${ }^{13,14}$, K. C. Gendreau ${ }^{14}$, C. Markwardt ${ }^{14}$, T. E. Strohmayer ${ }^{14}$, P. Uttley ${ }^{15}$, F. Tombesi ${ }^{13,14,16}$ and D.J.K. Buisson ${ }^{1}$

${ }^{1}$ School of Physics and Astronomy, University of Southampton, Southampton, SO17 1BJ, UK

${ }^{2}$ Kapteyn Astronomical Institute, University of Groningen, PO Box 800, NL-9700 AV Groningen, the Netherlands

${ }^{3}$ Instituto de Astrofísica de Canarias (IAC), Vía Láctea $s / n$, La Laguna 38205, S/C de Tenerife, Spain

${ }^{4}$ Departamento de Astrofísica, Universidad de La Laguna, La Laguna, E-38205, S/C de Tenerife, Spain

${ }^{5}$ MIT Kavli Institute for Astrophysics and Space Research, MIT, 70 Vassar Street, Cambridge, MA 02139, USA

${ }^{6}$ Consorcio de Investigación del Golfo de México, CICESE, Carretera Ensenada-Tijuana 3918, 22860 Ensenada, BC, Mexico

${ }^{7}$ Cahill Center for Astronomy and Astrophysics, California Institute of Technology, Pasadena, CA 91125, USA

${ }^{8}$ Center for Astrophysics | Harvard \& Smithsonian, 60 Garden St. Cambridge, MA 02138, USA

${ }^{9}$ Extreme Natural Phenomena RIKEN Hakubi Research Team, RIKEN Cluster for Pioneering Research, 2-1 Hirosawa, Wako, Saitama 351-0198, Japan

${ }^{10}$ The Hakubi Center for Advanced Research, Kyoto University, Kyoto 606-8302, Japan

${ }^{11}$ Eureka Scientific, Inc., 2452 Delmer Street, Oakland, CA 94602, USA

${ }^{12}$ SRON, Netherlands Institute for Space Research, Sorbonnelaan 2, 3584 CA Utrecht, The Netherlands

${ }^{13}$ Department of Astronomy, University of Maryland, College Park, MD 20742, USA

${ }^{14}$ Astrophysics Science Division, NASA Goddard Space Flight Center, Greenbelt, MD 20771, USA

${ }^{15}$ Anton Pannekoek Institute for Astronomy, University of Amsterdam, Science Park 904, 1098 XH Amsterdam, The Netherlands

${ }^{16}$ Department of Physics, University of Rome "Tor Vergata", Via della Ricerca Scientifica 1, I-00133 Rome, Italy

Accepted 2020 July 20. Received 2020 July 20; in original form 2020 April 8

\begin{abstract}
We present a detailed X-ray spectral and variability study of the full 2018 outburst of MAXI J1727-203 using NICER observations. The outburst lasted approximately four months. Spectral modelling in the $0.3-10 \mathrm{keV}$ band shows the presence of both a soft thermal and a hard Comptonised component. The analysis of these components shows that MAXI J1727-203 evolved through the soft, intermediate and hard spectral states during the outburst. We find that the soft (disc) component was detected throughout almost the entire outburst, with temperatures ranging from $\sim 0.4 \mathrm{keV}$, at the moment of maximum luminosity, to $\sim 0.1 \mathrm{keV}$ near the end of the outburst. The power spectrum in the hard and intermediate states shows broadband noise up to $20 \mathrm{~Hz}$, with no evidence of quasi-periodic oscillations. We also study the rms spectra of the broadband noise at $0.3-10 \mathrm{keV}$ of this source. We find that the fractional rms increases with energy in most of the outburst except during the hard state, where the fractional rms remains approximately constant with energy. We also find that, below $3 \mathrm{keV}$, the fractional rms follows the same trend generally observed at energies $>3 \mathrm{keV}$, a behaviour known from previous studies of black holes and neutron stars. The spectral and timing evolution of MAXI J1727-203, as parametrised by the hardness-intensity, hardness-rms, and rms-intensity diagrams, suggest that the system hosts a black hole, although we could not rule out a neutron star.
\end{abstract}

Key words: Accretion, accretion discs - black hole physics - X-rays: binaries stars: individual: MAXI J1727-203 contain a compact object, either a black hole $(\mathrm{BH})$ or 
a neutron star (NS) and an evolved low-mass companion star. LMXBs for which the compact object is a black-hole candidate are known as BH low-mass X-ray binaries (BH LMXBs). The energy spectra of BH LMXBs are characterised by two main components: a soft thermal component and a hard power-law like component (e.g. Remillard \& McClintock 2006; Belloni 2010). The thermal component is generally described by a multi-colour disc blackbody model (Mitsuda et al. 1984) peaking at 1-2 keV (see review by Done et al. 2007, and references therein) and thought to be produced by a geometrically thin and optically thick accretion disc (Shakura \& Sunyaev 1973). The hard component is thought to be produced by a region of hot plasma, around the compact object and the accretion disc (the socalled "corona"; Sunyaev \& Truemper 1979; Sunyaev \& Titarchuk 1980). A thermal Comptonisation model, in which high-energy photons are emitted by inverse Compton scattering (Sunyaev \& Titarchuk 1980), has been proposed to explain the hard component of the BH LMXBs energy spectra (e.g. Titarchuk 1994; Zdziarski \& Gierliński 2004; Done et al. 2007; Burke et al. 2017).

BH LMXBs show a variety of spectral and timing properties during an outburst (see e.g. van der Klis 1989; Méndez \& van der Klis 1997; van der Klis 2000; Homan \& Belloni 2005; Remillard \& McClintock 2006; Belloni 2010; Belloni et al. 2011; Plant et al. 2014; Motta 2016). Two main spectral states can be defined (see e.g., Tanaka 1989; van der Klis 1994): the low/hard state (LHS), when the thermal Comptonised component dominates the energy spectrum, and the high/soft state (HSS) when the thermal component dominates the spectrum. In the LHS, however, a multi-colour disc blackbody component can be detected (e.g. Capitanio et al. 2009; Wang-Ji et al. 2018). In this state, the powerdensity spectrum (PDS) is characterised by a strong broadband noise component with a fractional $\mathrm{rms}$ amplitude of 30\%-50\% (e.g. Méndez \& van der Klis 1997; Belloni et al. 2005; Remillard \& McClintock 2006; Muñoz-Darias et al. 2011; Motta 2016). In addition, quasi-periodic oscillations (QPOs) of type-C can be detected (e.g., Casella et al. 2004; Belloni et al. 2005). These oscillations have a centroid frequency ranging from $0.01 \mathrm{~Hz}$ to $30 \mathrm{~Hz}$. In the HSS, a weak power-law component is sometimes detected in the energy spectrum (e.g. Capitanio et al. 2009). The broadband fractional rms of $\mathrm{BHs}$ in this state is generally less than $5 \%$ (Méndez \& van der Klis 1997), and QPOs are sometimes detected, too (e.g. Remillard \& McClintock 2006; MuñozDarias et al. 2011; Motta 2016).

Between the LHS and HSS, two intermediate states can be distinguished in terms of variability: the hard intermediate state (HIMS) and the soft intermediate state (SIMS) (see, e.g. Homan \& Belloni 2005; Belloni 2010). The HIMS shows less broadband fractional rms than the hard state (10\%-30\%; e.g. Muñoz-Darias et al. 2011; Motta et al. 2012) and type-C QPOs can be present (e.g. Casella et al. 2004; Belloni et al. 2005; Belloni \& Stella 2014). The SIMS is characterised by a weak power-law noise component that replaces the broadband noise component present in the HIMS, and type-A or type-B QPOs (e.g. Wijnands et al. 1999; Homan et al. 2001; Casella et al. 2004; Belloni et al. 2005; Belloni \& Stella 2014). Type-B QPOs have centroid frequencies in the $1-7 \mathrm{~Hz}$ frequency range (Gao et al. 2017) and a quality factor, $\mathrm{Q}>6$. Type-A QPOs have centroid frequencies in the $6.5-8 \mathrm{~Hz}$ frequency range and are broader than type- $\mathrm{B}$ and type-C QPOs, with a quality factor of $\mathrm{Q}=1-3$ (Wijnands et al. 1999; Casella et al. 2004; Belloni \& Stella 2014).

The evolution of a BH LMXB through an outburst can be well illustrated using the hardness-intensity diagram (HID; e.g. Homan et al. 2001; Remillard \& McClintock 2006; Belloni et al. 2006). At the beginning of the outburst, the source is in the LHS and its intensity increases at approximately constant hardness ratio, drawing a vertical line in the right part of the HID. At some point, in the outburst evolution, the source starts a transition to the HSS, moving to the left in the diagram at an approximately constant luminosity. This transition corresponds to the top horizontal branch in the HID (HIMS and SIMS), reaching the HSS at the top left part of the HID. During the HSS, the source starts to decrease its intensity, moving down in the diagram. Eventually, the source returns to the HIMS and SIMS, drawing a horizontal branch in the HID, but in the opposite direction, from left to right. Before the end of the outburst, the source reaches the hard state again, to finally return to quiescence. This very particular pattern in the HID of BH LMXBs is known as the q-track and it is often discussed in terms of hysteresis (e.g., Miyamoto et al. 1995). Multiple outbursts of different sources follow this q-track: e.g. XTE 1550-564, GX 339-4, H1743-322 and GRO J1655-40 (Homan et al. 2001; Belloni et al. 2005; Fender et al. 2009; Dunn et al. 2010; Uttley \& Klein-Wolt 2015).

The outburst evolution can also be analysed using the hardness-rms diagram (HRD, Belloni et al. 2005) and the rms-intensity diagram (RID, Muñoz-Darias et al. 2011). The different spectral states show different fractional rmshardness ratio correlations. Observations in the LHS are located on the top right side of the HRD. When the source enters the HIMS and the SIMS, it moves to the bottom left side of the HRD diagonally until the source reaches the HSS. Finally, the evolution reverses, returning to the HIMS and the SIMS following the same track as before, until it reaches the hard state again at the top right side of the HRD. The evolution in the RID is counterclockwise, similar to the one observed in the HID. In the LHS, the source evolves along a diagonal line from the bottom left to the top right of the diagram. This line is called the "Hard Line" (HL, Muñoz-Darias et al. 2011). When it makes the transition to the HIMS and SIMS, the source moves horizontally to the left side of the RID. Then the source reaches the HSS and starts to move down along a diagonal following the $1 \% \mathrm{rms}$ line. Finally, the source returns to the HIMS and SIMS moving horizontally to the right side of the diagram. At some point the source reaches again the $30 \% \mathrm{rms}$ line and goes down diagonally following the so-called "Adjacent Hard Line" (AHL), which is coincident to the Hard Line.

LMXBs in which the compact object is a NS are known as NS LMXBs. The energy spectra of NS LMXBs are characterised by three components: a disc blackbody component and a Comptonised component as for BH LMXBs, and a blackbody component from the emission of the surface of the NS and its boundary layer (e.g. Mitsuda et al. 1984; Di Salvo et al. 2000; Gierliński \& Done 2002; Lin et al. 2007). NS LMXBs show different X-ray spectral states (for a review, see van der Klis 2006). At high accretion rates, NS LMXBs follow Z-tracks in the HID and the colour-colour diagrams. These sources are known as $\mathrm{Z}$ sources. At low ac- 
cretion rates, NS LMXBs are known as atoll sources due to the tracks they follow in colour-colour diagrams (Hasinger \& van der Klis 1989). Atoll sources show three X-ray spectral states that are comparable to the X-ray spectral states of $\mathrm{BH}$ LMXBs (e.g. van der Klis 2006; Muñoz-Darias et al. 2014). Besides, the hysteresis observed in BH LMXBs has been also observed in NS LMXBs (Muñoz-Darias et al. 2014), sometimes even the q-track (Körding et al. 2008).

Some differences between BH LMXBs and NS LMXBs have been observed in the X-ray spectral and timing properties. The hard state of NS systems is softer than that of $\mathrm{BH}$ systems (e.g. Done \& Gierliński 2003). In terms of timing properties, the most important difference between the two types of LMXBs is the presence of kilo-hertz QPOs $(\mathrm{kHz}$ QPOs) at frequencies between $300 \mathrm{~Hz}$ and $1.2 \mathrm{kHz}$ for NS (van der Klis 2006; van Doesburgh et al. 2018). In terms of the broadband noise component and low-frequency QPOs (LFQPOs), NSs and BHs systems can be very similar (e.g. Klein-Wolt \& van der Klis 2008) but, while BH LMXBs usually show broadband noise up to $500 \mathrm{~Hz}$, NS systems can show broadband noise at higher frequencies (Sunyaev \& Revnivtsev 2000).

MAXI J1727-203 was discovered on 2018 June 5 with MAXI/GSC (Yoneyama et al. 2018). Ludlam et al. (2018b) and Kennea et al. (2018) reported, respectively, observations performed the same day with the Neutron star Interior Composition Explorer (NICER; Gendreau et al. 2012) and with the Neil Gehrels Swift Observatory (Swift; Gehrels et al. 2004). A hard-to-soft state transition and the disc properties of the system in the soft state, led to the possible identification of the source as a $\mathrm{BH}$ transient (Negoro et al. 2018). In mid-July of 2018, a soft-to-hard transition was observed with Swift/XRT (Tomsick et al. 2018).

NICER (Gendreau et al. 2012) is an X-ray instrument aboard the International Space Station (ISS) launched in 2017. It consists on 52 functioning detectors. Photons in the $0.2-12 \mathrm{keV}$ energy band can be detected to a time resolution of 300 ns. In this paper, we present the first study of the spectral and timing evolution of the 4-months long outburst of MAXI J1727-203 as observed with NICER. In Section 2 we describe the observations and data analysis. In section 3 we present the results of the spectral and timing study. In section 3.1 we describe the outburst evolution. In sections 3.2 and 3.3 we describe the timing and spectral properties, respectively. Finally, in Section 4 we discuss the nature of the compact object of the source and the identification of its spectral states.

\section{OBSERVATION AND DATA ANALYSIS}

NICER observed MAXI J1727-203 86 times between 2018 June 5 and 2018 October 7 (ObsID 1200220101 1200220186). The data were analysed using the software HEASOFT version 6.26 and NICERDAS version 6.0. The latest CALDB version 20190516 was used. We applied standard filtering and cleaning criteria, including the data where the pointing offset was $<54^{\prime \prime}$, the dark Earth limb angle was $>15^{\circ}$, the bright Earth limb angle was $>30^{\circ}$, and the International Space Station (ISS) was outside the South Atlantic Anomaly (SAA). We removed data from detectors 14 and 34 which occasionally show episodes of increased elec- tronic noise, so all our results are based on using NICER's 50 other active detectors. Also, we excluded time intervals showing strong background flare-ups, that is, time intervals with an averaged count rate in the $13-15 \mathrm{keV}$ energy band higher than $1 \mathrm{c} / \mathrm{s}$. The good time intervals (GTIs) of each observation were separated into several data segments (1-9) based on the orbit of the ISS. The background was calculated using the "3C50_RGv5" model provided by the NICER team.

To create the long-term light curve and the HID of the outburst, we first produced 1-s binned light curves in the $0.5-12 \mathrm{keV}, 2-3.5 \mathrm{keV}$ and $6-12 \mathrm{keV}$ energy bands for each data segments using XSELECT. We then applied the background correction for each light curve and calculated averages per data segment. We defined intensity as the average count rate in the $0.5-12 \mathrm{keV}$ energy range and the hardness ratio as the ratio between the $6-12 \mathrm{keV}$ and the $2-3.5 \mathrm{keV}$ band count rates (both background subtracted).

We extracted a background-subtracted energy spectra for each data segment using the "3C50_RGv5" model mentioned above. We fitted the energy spectra of MAXI J1727203 in the energy band $0.3-10 \mathrm{keV}$ using XSPEC (V. 12.10.1; Arnaud 1996). We rebinned the spectra by a factor of 3 to correct for energy oversampling and then to have at least 25 counts per bin. In addition, we added a systematic error of $1 \%$ in the energy range $2-10 \mathrm{keV}$ (suggested by the NICER team). We found strong instrumental residuals below $2 \mathrm{keV}$. These residuals are typical for X-ray missions and Si-based detectors (e.g. Ludlam et al. 2018a; Miller et al. 2018). We therefore added a 5\% systematic error in the $0.3-2 \mathrm{keV}$ energy band (also suggested by the NICER team). We fitted the energy spectra with an absorbed (TBABS in XSPEC, Wilms et al. 2000) powerlaw model, TBABSXPOWERLAW, an absorbed disc blackbody (Mitsuda et al. 1984), TBABS×DISKBB, and an absorbed combination of a thermally Comptonisation model (Zdziarski et al. 1996; Życki et al. 1999) and a multi-colour disc blackbody TBABS $\times($ NTHCOMP+DISKBB $)$. Fitting the spectra with the models TBABS $\times$ POWERLAW and TBABSXDISKBB did not give satisfactory fits in terms of $\chi^{2} / d o f$ and expected spectral parameters. Therefore, in this paper, we only report the results of using the model TBABS $\times$ (NTHCOMP+DISKBB). In order to obtain the fluxes of the different components, we added two CFLux components to the models. The solar abundances were set according to Wilms et al. (2000) and the hydrogen column density $\left(N_{\mathrm{H}}\right)$ of the TBABs was left free. The cross section was set according to Verner et al. (1996). The $1 \sigma$ errors of the parameters were calculated from a Markov Chain Monte Carlo of length 10000 with a 2000step burn-in phase.

For the Fourier timing analysis, we constructed Leahynormalized power spectra (Leahy et al. 1983) using data segments of 131 seconds and a time resolution of $125 \mu \mathrm{s}$. The minimum frequency was $0.007 \mathrm{~Hz}$ and the Nyquist frequency was $4096 \mathrm{~Hz}$. Then we averaged the power spectra per observation and subtracted the Poisson noise based on the average power in the $3-4 \mathrm{kHz}$ frequency range. Finally, we converted the power spectra to squared fractional rms (van der Klis 1995). We obtained the integrated fractional rms amplitude from $0.01 \mathrm{~Hz}$ to $64 \mathrm{~Hz}$. To obtain the rms spectrum (i.e. fractional rms amplitude vs energy), we repeated the previous procedure for the following energy bands: $0.3-0.8 \mathrm{keV}$, 
$0.8-2.0 \mathrm{keV}, 2.0-5.0 \mathrm{keV}$ and $5.0-12 \mathrm{keV}$. We obtained the $0.01-64 \mathrm{~Hz}$ fractional rms amplitude for all these bands and plotted the fractional rms amplitude vs energy to study the evolution of the energy dependence of the fractional rms amplitude.

To fit the power spectra we used a multi-Lorentzian function: the sum of several Lorentzians. We give the frequency of the Lorentzians in terms of the characteristic frequency, which is the frequency where the component contributes most of its variance per logarithmic interval of frequency (Belloni et al. 2002): $v_{\max }=\sqrt{v_{0}+(F W H M / 2)^{2}}=$ $v_{0} \sqrt{1+1 / 4 Q^{2}}$. The quality factor $Q$ is defined as $Q=$ $v_{0} / F W H M$, where $F W H M$ is the full width at half maximum and $v_{0}$ the centroid frequency of the Lorentzian.

\section{RESULTS}

\subsection{Outburst evolution}

We show the NICER light curve of the 2018 outburst of MAXI J1727-203, which covers a period of 123 days, in the $0.5-12 \mathrm{keV}$ energy band in the top panel of Figure 1. Based on our spectral and variability studies, which are described below, we define four phases of the outburst in Figure 1: phase A (from MJD 58274 to MJD 58278; shown with orange diamonds), phase B (from MJD 58278 to MJD 58296; shown with blue triangles), phase C (from MJD 58296 to MJD 58322; shown with black circles) and phase D (from MJD 58322 to the end of the outburst; shown with red filled crosses).

The first NICER detection of the source was on MJD 58274 , at a count rate of $\sim 1400 \mathrm{c} / \mathrm{s}$, indicating that the NICER observations caught the outburst already at a high flux. The intensity increased very quickly until MJD 58279 in phase A when the source reached a maximum intensity of $\sim 5960 \mathrm{c} / \mathrm{s}$. After the maximum, the flux decreased monotonically, although the decay can be divided into 3 parts. As the source entered phase B of the outburst, its intensity decreased smoothly from $\sim 5960 \mathrm{c} / \mathrm{s}$ to $\sim 3400 \mathrm{c} / \mathrm{s}$ over the next 18 days of observation. During phase $\mathrm{C}$ of the outburst, the intensity decreased faster than in phase $\mathrm{B}$, from $\sim 2500 \mathrm{c} / \mathrm{s}$ to $\sim 300 \mathrm{c} / \mathrm{s}$ over 26 days. Finally, in phase D the intensity decreased from $\sim 90 \mathrm{c} / \mathrm{s}$ to $\sim 4 \mathrm{c} / \mathrm{s}$ over the last 75 days of X-ray monitoring. After that, the apparent position of the source was located behind the Sun from the point of view of NICER in its Earth orbit on-board the ISS. After the MAXI J1727-203 occultation by the Sun, NICER did not perform further observations of this source.

We also show the $2-10 \mathrm{keV} M A X I^{1}$ and the $15-50 \mathrm{keV}$ Swift/BAT ${ }^{2}$ light curves of MAXI J1727-203 in the second and third panels of Figure 1, respectively. The rise of the outburst was detected by $M A X I$, showing that the intensity increased by a factor of $\sim 50$ in four days. In phase B, the MAXI intensity decayed faster than the NICER intensity. The analysis of the $2-10 \mathrm{keV} \mathrm{NICER} \mathrm{light} \mathrm{curve} \mathrm{shows} \mathrm{the}$ same trend as the $0.5-12 \mathrm{keV}$ light curve, probably indicating that the difference between NICER and MAXI is due to

\footnotetext{
1 http://maxi.riken.jp/pubdata/v6m/J1728-203/index.html

2 https://swift.gsfc.nasa.gov/results/transients/weak/MAXIJ1727203.lc.txt
}

differences in their respective effective areas. In phases $\mathrm{C}$ and $\mathrm{D}$ of the outburst, the evolution of both NICER and MAXI light curves were similar. The Swift/BAT light curve did not sample the rise of the outburst, however, it gives additional information during phases $\mathrm{B}$ and $\mathrm{C}$, where the $15-50 \mathrm{keV}$ intensity showed a bump. In phases $\mathrm{C}$ and $\mathrm{D}$, the Swift/BAT intensity decayed until the end of the outburst.

In the fourth panel in Figure 1 we show the temporal evolution of the hardness ratio (as estimated from NICER data) during the whole outburst. The different phases of the outburst show a different behaviour of the hardness ratio. In phase A of the outburst, the hardness ratio drop from $\sim 0.05$ to $\sim 0.02$. In phase B the hardness ratio remained constant with values around $\sim 0.004$. In phase $\mathrm{C}$ the hardness ratio increased from $\sim 0.03$ to $\sim 0.09$. In phase $\mathrm{D}$ the source showed an approximately constant hardness ratio with an average value of $\sim 0.09$. Due to the data-gaps between phases B and $\mathrm{C}$, and phases $\mathrm{C}$ and $\mathrm{D}$, we arbitrarily chose the limits between phases in the middle of the gap.

In the top panel of Figure 2 we show the HID. The first point is marked with a green star in the phase A of the outburst (orange diamonds). During this phase, the source evolved in the top part of the HID from the right side to the left side. In the phase B of the outburst (blue triangles) the source reduced its intensity at an approximately constant hardness ratio. Then the source entered the phase $\mathrm{C}$ of the outburst (black circles) and evolved from the top left to the right side of the diagram. Finally, during the phase D of the outburst (red crosses) the source evolved to the bottom right side of the diagram. Although we are missing the rise of the outburst, a q-track shape is clear in Figure 2.

\subsection{Timing properties}

As expected from LMXBs in outburst, the X-ray variability of MAXI J1727-203 also evolved through the 2018 outburst. The bottom panel in Figure 1 shows the temporal evolution of the averaged fractional rms amplitude. During the first observation (MJD 58274) MAXI J1727-203 showed a fractional rms amplitude of $\sim 12 \%$. Then, the fractional rms decreased down to $\sim 3 \%$ in phase A of the outburst. In phase $\mathrm{B}$, the fractional $\mathrm{rms}$ amplitude ranged from $\sim 2 \%$ to $\sim 0.5 \%$, in phase $\mathrm{C}$ it increased from $\sim 7 \%$ to $\sim 27 \%$ and, in phase $\mathrm{D}$, it remained approximately constant at $\sim 30 \%$.

In the bottom panel of Figure 2 we show the HRD. The first observation is marked with a green star and it showed a hardness ratio of $\sim 0.05$ and a fractional $\mathrm{rms}$ amplitude of $\sim 12 \%$. Then, the source evolved to the bottom left part of the diagram reaching values of the fractional $\mathrm{rms}$ amplitude $<1 \%$. Finally, the evolution reversed and the source moved to the top right side of the diagram, increasing its hardness ratio and the fractional rms amplitude.

Figure 3 shows how MAXI J1727-203 evolves in the absolute rms-intensity diagram. The source described the anticlockwise pattern that has been observed for other BHs (e.g. MAXI J1348-630, Zhang et al. in prep; GX 339-4, MuñozDarias et al. 2011). The first point of the outburst in the RID is denoted with a green star. As the source evolved it crossed the $10 \%$ fractional rms amplitude line increasing its intensity. Two days later, on MJD 58276, the source crossed the $5 \%$ line and after that it moved horizontally to the left of the diagram. From MJD 58278 to MJD 58294, 

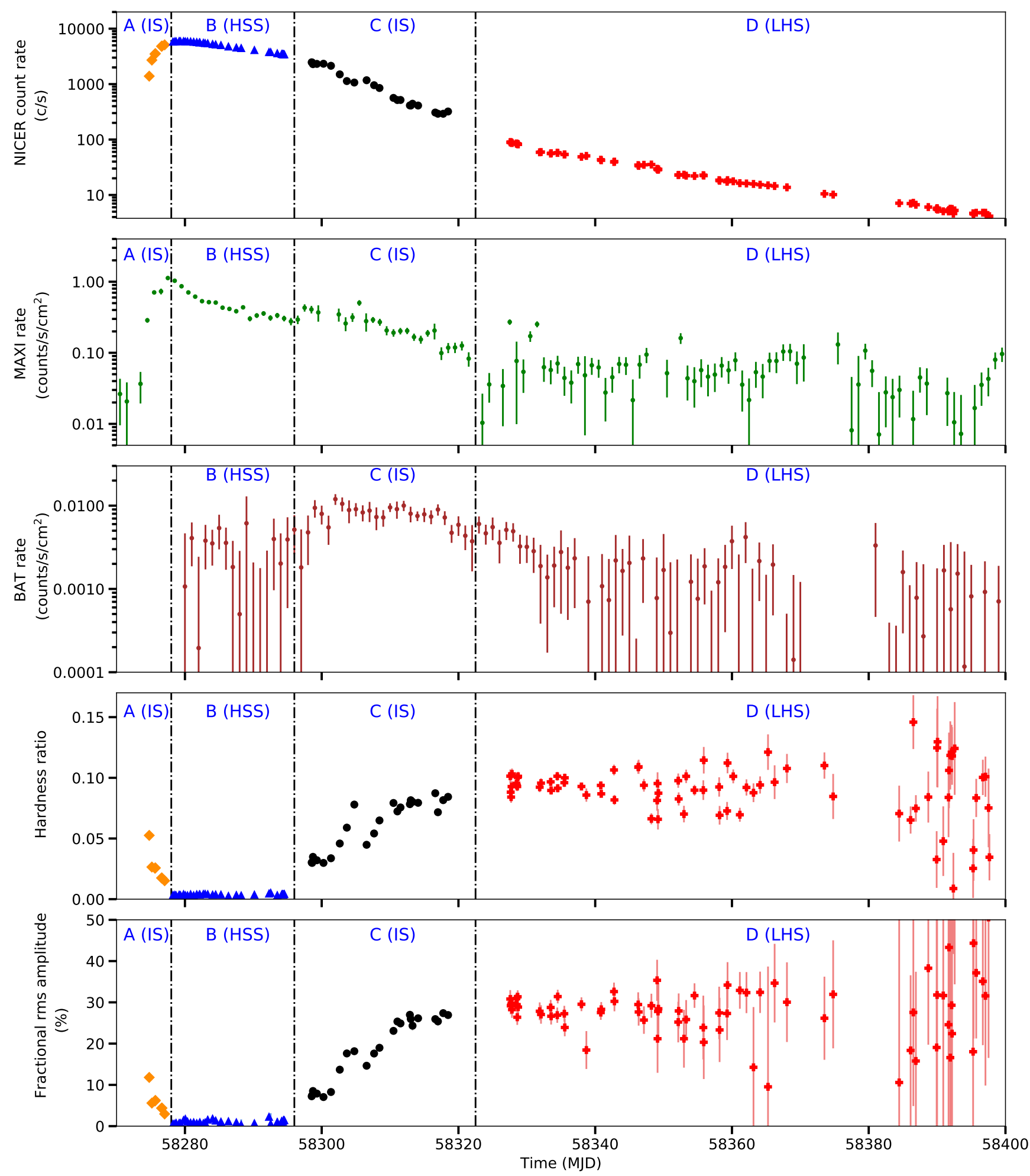

Figure 1. Top panel: NICER light curve of the 2018 outburst of MAXI J1727-203 in the $0.5-12 \mathrm{keV}$ energy band. Second panel: $M A X I$ light curve of the 2018 outburst of MAXI J1727-203 in the 2-10 keV energy band. Third panel: Swift/BAT light curve in the $15-50$ $\mathrm{keV}$ energy band. Fourth panel: Temporal evolution of the hardness ratio $(6-12 \mathrm{keV}) /(2-3.5 \mathrm{keV})$. Bottom panel: Temporal evolution of the $0.01-64 \mathrm{~Hz}$ fractional rms amplitude in the $0.5-12 \mathrm{keV}$ energy band. Colours and symbols in the NICER light curve, hardness ratio and fractional rms amplitude represent different phases of the outburst. Orange diamonds: Phase A. Blue triangles: Phase B. Black circles: Phase C. Red filled crosses: Phase D. The dotted dashed lines divide the four phases (see section 4 for a physical interpretation of these intervals). 

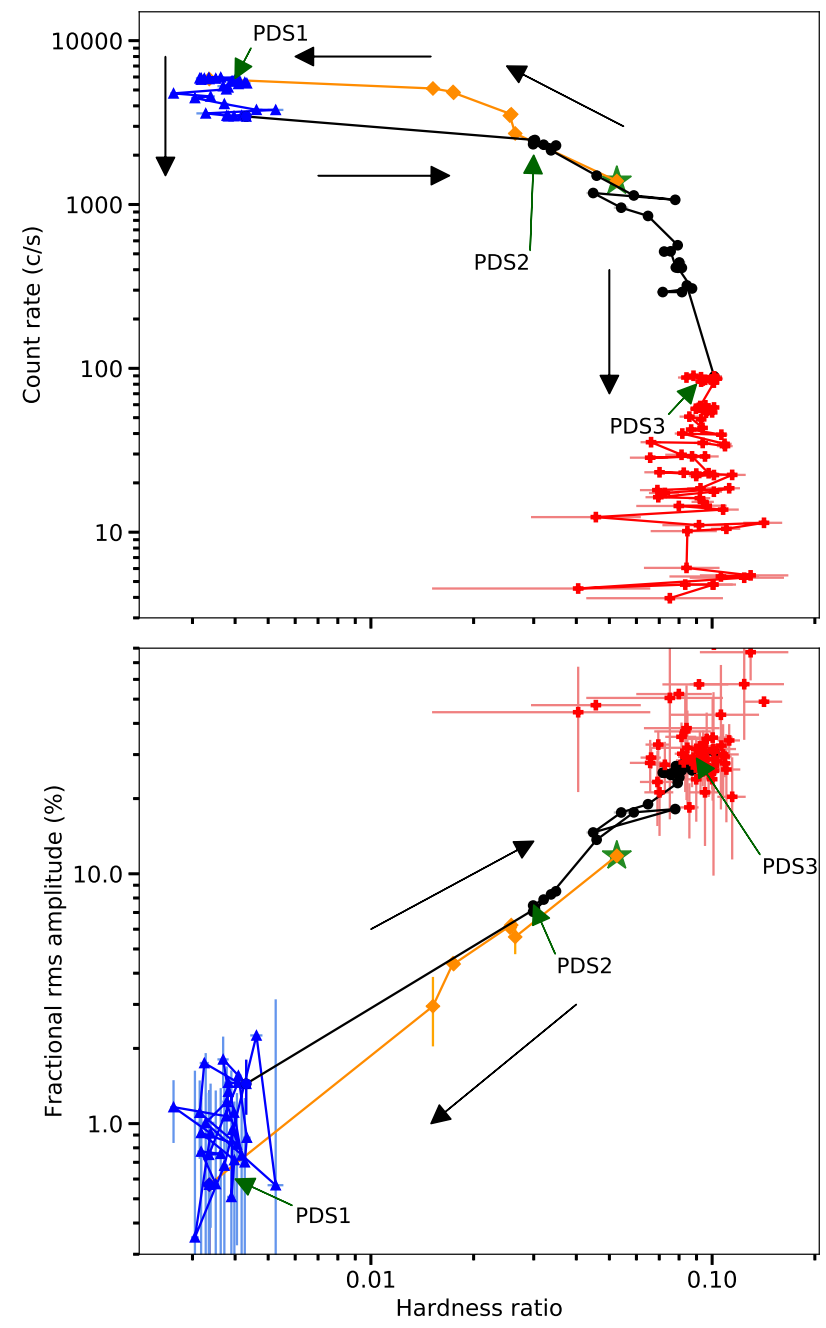

Figure 2. Top panel: HID of MAXI J1727-203 during the 2018 outburst. The hardness ratio is defined as the count-ratio in the bands $(6-12 \mathrm{keV}) /(2.0-3.5 \mathrm{keV})$. The count rate is obtained in the $0.5-12 \mathrm{keV}$ energy band. Bottom panel: HRD of MAXI J1727203 during the 2018 outburst. The fractional rms amplitude is obtained in the $0.01-64 \mathrm{~Hz}$ frequency range and $0.5-12 \mathrm{keV}$ energy band. The colours are the same as in Figure 1. The green star marks the first observation. Black arrows indicate the direction of the temporal evolution through the diagram. "PDS1", "PDS2" and "PDS3" mark the location in the plots of the data used to create the representative power spectra shown in Figure 4. Colours and symbols are the same as described in Figure 1.

MAXI J1727-203 stayed close to the $1 \%$ rms line while the intensity decreased. This corresponds to the softest part of the HID and the bottom-left part of the HRD (shown with blue triangles in the bottom panel of Figure 1 and Figure 2). After MJD 58298 the source evolved in the opposite way going back to the right side of the RID. On MJD 58302, MAXI J1727-203 crossed the 10\% rms line and on MJD 58310 , it crossed the $20 \%$ rms line. 17 days later, on MJD 58327 , the source evolved around the $30 \%$ rms line, identifying this as the Adjacent Hard Line. This is shown with red filled crosses in Figure 1 and Figure 2.

Figure 4 shows three representative examples of the

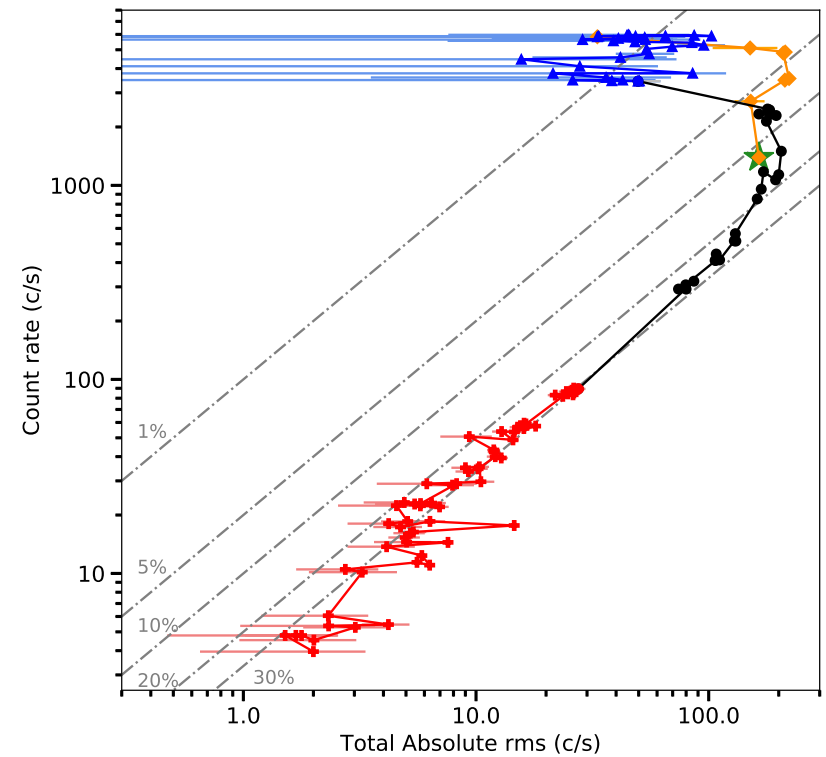

Figure 3. RID of MAXI J1727-203 during the 2018 outburst. The total absolute rms is obtained in the $0.01-64 \mathrm{~Hz}$ frequency range and $0.5-12 \mathrm{keV}$ energy band. The count rate is obtained in the $0.5-12 \mathrm{keV}$ energy band. The dashed lines represent the lines of constant fractional rms amplitude. Colours and symbols are the same as in Figure 1. The green star marks the first NICER observation.

NICER PDS at three different stages of the outburst (marked in the HID and the RID as "PDS1", "PDS2" and "PDS3", respectively). The PDS of the observations before MJD 58278 (phase A of the outburst) showed a significant broadband noise component up to $\sim 20 \mathrm{~Hz}$ and no significant QPOs (e.g. panel (ii) in Figure 4). In phase B, the PDS of all the observations revealed little to no significant variability (e.g. panel (i) in Figure 4). This corresponds to the interval plotted with blue triangles of the bottom panel of Figure 1 and the HID and RID. Then, from MJD 58298 to the end of the outburst (phases C and D), a broadband noise component was present with similar power-spectral shape as that in panel (ii) and panel (iii) in Figure 4. In this period, there was significant broadband noise extending up to a frequency of $\sim 20 \mathrm{~Hz}$ on MJD 58298; after this date the maximum frequency of this broadband noise component decreased down to hundredths of $\mathrm{Hz}$ as the source evolved towards the end of the outburst. Figure 5 shows the evolution of the characteristic frequency of the broadband noise component with intensity. We found that they are correlated.

We searched for QPOs in the PDS of MAXI J1727203 in the $0.5-12 \mathrm{keV}$ and the $2-12 \mathrm{keV}$ energy bands per observation, per orbit and per region of the HID. We found evidence for QPOs in four cases: at $0.2 \mathrm{~Hz}(10.0 \pm 1.6 \%$ rms, ObsID 1200220134), $0.5 \mathrm{~Hz}(7.3 \pm 0.7 \%$ RMS, ObsID $1200220127), 3 \mathrm{~Hz}(6.1 \pm 0.9 \% \mathrm{rms}$, ObsID 1200220131) and $6 \mathrm{~Hz}(1.9 \pm 0.2 \% \mathrm{rms}$, ObsID 1200220102). These QPOs are all between 3 and $3.5 \sigma$ significant single trial. When considering the number of trials, these QPOs are $<1 \sigma$ significant; however the fractional rms amplitude we measured serve as an indication of our sensitivity to detect QPOs.

Figure 6 shows the $0.01-64 \mathrm{~Hz}$ fractional rms spectrum 


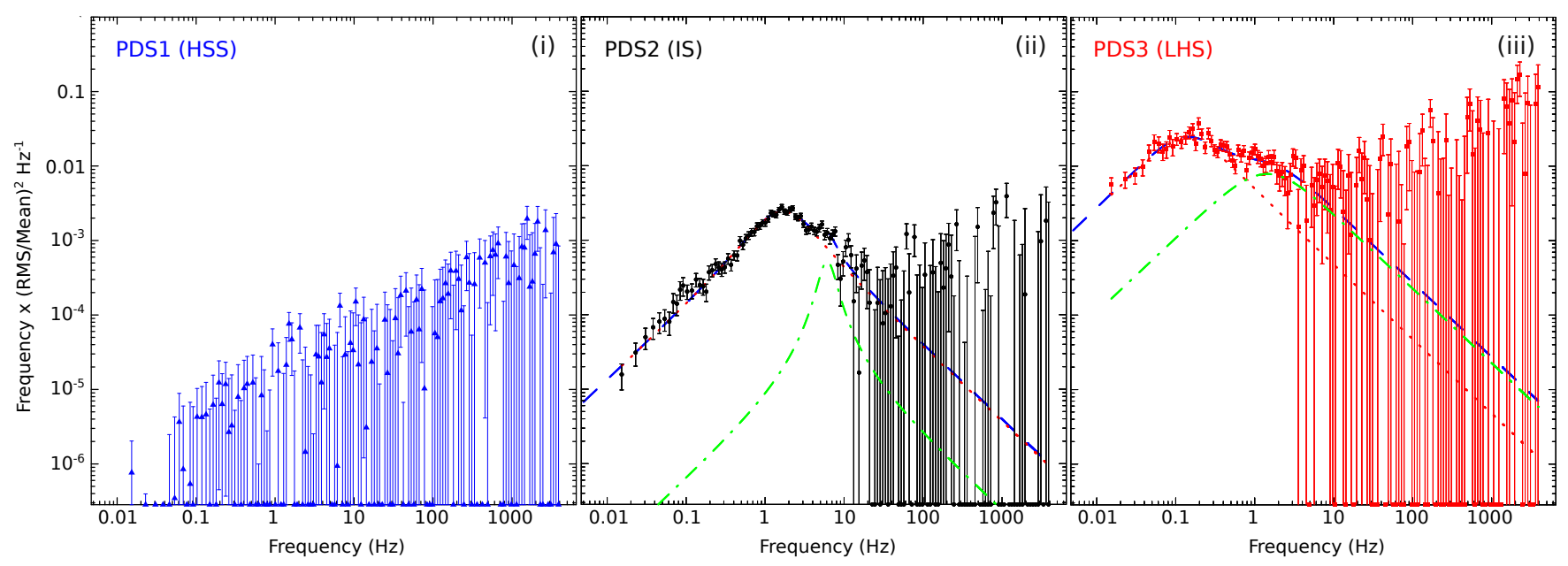

Figure 4. Three representative power spectra of the 2018 outburst of MAXI J1727-203. Panel (i) shows the power spectra of ObsID 1200220105. Panel (ii) shows the power spectra of ObsID 1200220120. Panel (iii) shows the power spectra of ObsID 1200220141. These observations occurred during phases B and C, respectively, of the outburst evolution. Dashed and dotted lines represent the best fit Lorentzians.

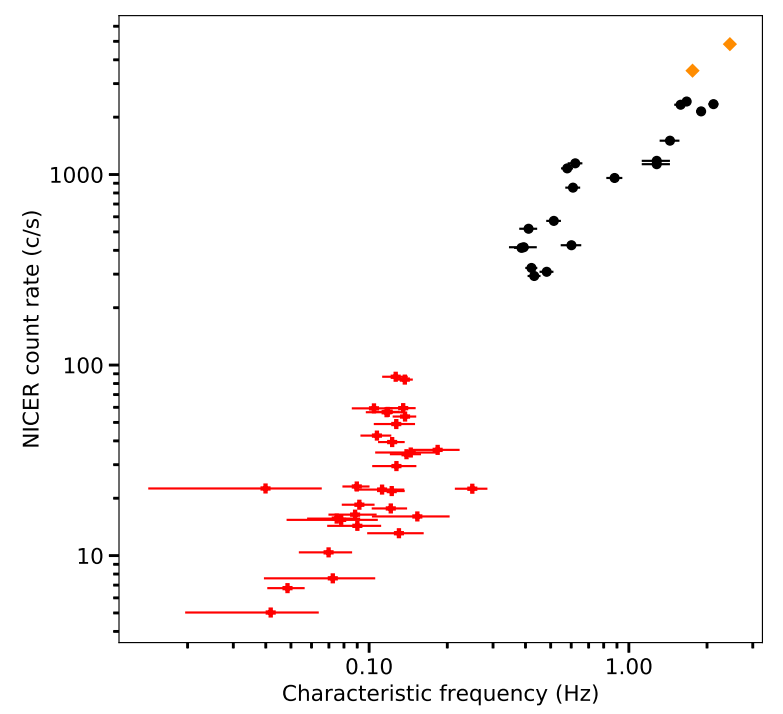

Figure 5. Characteristic frequency vs NICER count rate in the $0.5-12 \mathrm{keV}$ energy band. Colours and symbols correspond to the phases of the outburst as described in Figure 1.

of representative observations through the whole outburst in the $0.3-12 \mathrm{keV}$ energy band. The panels are chronologically ordered. The rms spectrum of panel (f) was made combining all the observations from MJD 58327 to MJD 58346 for the same reason. From panel (a) to panel (e) the fractional rms amplitude increased with energy. On panel (f) the fractional rms amplitude remained approximately constant with energy. During phase B of the outburst, the X-ray variability is very low, of the order of $\sim 1 \%$ fractional rms. We do not show the data of this phase in Figure 6 as we only obtain upper limits in the different energy bands.

\subsection{Spectral properties}

We fitted the energy spectra using the model TBABS $\times($ NTHCOMP+DISKBB $)$. First, we fitted all the energy spectra separately linking the $k T_{\text {seed }}$ parameter of NTHCOMP and $k T_{i n}$ of DISKBB, and we found that the electron temperature, $k T_{e}$, in NTHCOMP was always above the maximum energy of the instrument. Therefore, we fixed $k T_{e}$ at $1000 \mathrm{keV}$. Besides, we noted that the value of $N_{\mathrm{H}}$ in all the fitted energy spectra was consistent within errors. Therefore, we decided to link this parameter among all the spectra and to repeat the fitting. We obtained an average $N_{\mathrm{H}}$ of $(0.437 \pm 0.001) \times 10^{22} \mathrm{~cm}^{-2}$, and a relatively good fit, with a $\chi^{2} / d o f$ of 1.16 , for 11107 degrees of freedom (for a total of 60 spectra). The excess in $\chi^{2}$ is given by the fit to some spectra where the $5 \%$ of systematic errors below $2 \mathrm{keV}$ were not sufficient to mitigate the effect of instrumental residuals below $2 \mathrm{keV}$.

Figure 7 shows four representative spectra of each phase of the outburst. The best-fitting parameters are given in table A1, the evolution of the parameters is shown in Figure 8. In the upper panel of Figure 8 we show the temporal evolution of the total observed flux. Naturally, we observed the same trend as in the upper panel of Figure 1. In the second panel of Figure 8 we show the temporal evolution of the Comptonised component unabsorbed flux in the $0.3-10 \mathrm{keV}$ energy band. The third panel shows the contribution of the Comptonised component to the unabsorbed flux in per cent. Finally, in the last two panels, we show the temporal evolution of the photon index, $\Gamma$, of the Comptonised component and the inner disc temperature, $k T_{i n}$, of the disc component.

The phases identified in Figure 1 show different spectral behaviour as well, as it is shown in Figure 8. In phase A, the contribution of the Comptonised component was $\sim 20 \%$. The photon index ranged from $\sim 2.5$ to $\sim 2.7$ and the disc temperature was close to $\sim 0.4 \mathrm{keV}$. The flux of the Comptonised component was $\sim 30.0 \times 10^{-10} \mathrm{erg} \mathrm{cm}^{-2} \mathrm{~s}^{-1}$. The flux of the disc component, on the other hand, ranged from $\sim 95 \times 10^{-10}$ erg $\mathrm{cm}^{-2} \mathrm{~s}^{-1}$ to $\sim 135 \times 10^{-10} \mathrm{erg} \mathrm{cm}^{-2} \mathrm{~s}^{-1}$. This phase corre- 

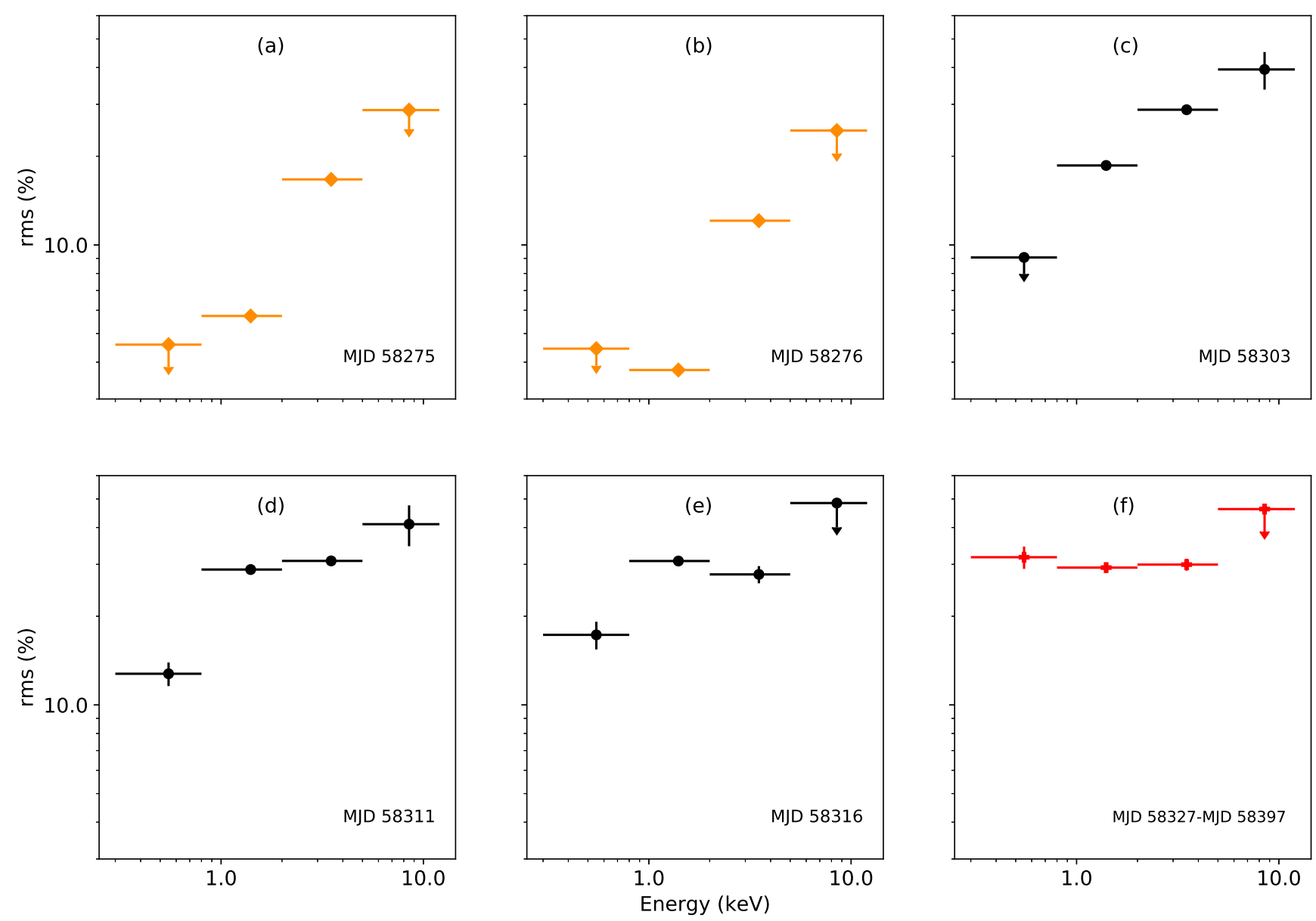

Figure 6. Evolution of the $0.01-64 \mathrm{~Hz}$ fractional rms spectrum of MAXI J1727-203. Plots are chronologically ordered. Colours and symbols correspond to the phases of the outburst as described in Figure 1. Arrows represent upper limits with a confidence of $3 \sigma$.

sponds to the regions with orange diamonds in Figures 2 and 3. In phase $\mathrm{B}$ the Comptonised flux dropped to $\sim 8 \times 10^{-10}$ erg $\mathrm{cm}^{-2} \mathrm{~s}^{-1}$ and decreased until $\sim 4 \times 10^{-10}$ erg $\mathrm{cm}^{-2} \mathrm{~s}^{-1}$ on MJD 58294 and, as a consequence, the contribution of the Comptonised component decreased to $\sim 4 \%$. The photon index varied from $\sim 2.7$ to $\sim 3.1$ and the disc temperature decreased from $\sim 0.45$ to $\sim 0.3 \mathrm{keV}$. This phase corresponds to the region plotted with blue triangles in the HID and the interval with lower fractional rms amplitude in the bottom panel of Figure 1 and Figure 3 (also plotted with blue triangles on the RID). In phase $\mathrm{C}$ the contribution of the Comptonised component was higher than in the previous phase. At the beginning of the phase, the contribution of the Comptonised component was $\sim 25 \%$ and increased up to $\sim 40 \%$. The photon index and the disc temperature decreased from $\sim 2.5$ to $\sim 2.0$ and from $\sim 0.3 \mathrm{keV}$ to $\sim 0.15 \mathrm{keV}$, respectively. This region corresponds to the black circles in the HID and the RID, where the hardness ratio and the fractional rms amplitude increased again. Finally, in phase D, the contribution of the Comptonised component to the total unabsorbed flux was higher than $80 \%$, with the disc component becoming insignificant (i.e. not statistically required) after MJD 58342. The photon index and the disc temperature remained approximately constant around $\sim 1.8$ and $\sim 0.1 \mathrm{keV}$, respectively. This phase corresponds to the red filled crosses of the HID and the RID. In these phases, the hardness ratio and the fractional rms amplitude remained constant at their highest values.

Our spectral modelling did not require the addition of a line-component in the in the $6-7 \mathrm{keV}$ region. The addition of a Gaussian in this energy range led to non-physical results (the Gaussian component became too broad and the NTHCOMP component changed). If the sigma parameter of the line was fixed to the arbitrary value of 0.3 , we found that in some cases there was a significant line. In phases $\mathrm{A}$ and $\mathrm{C}$ we could find emission lines at $\sim 6.5 \mathrm{keV}$ at a significance of no more than $4 \sigma$ and an equivalent width of $\sim 0.09$ $\mathrm{keV}$. After averaging all data of phase D in the period MJD 58327-58340, we were able to find a $\sim 3 \sigma$ emission line at $\sim 6.5 \mathrm{keV}$ and an equivalent width of $\sim 0.05 \mathrm{keV}$ (in this case the sigma parameter was also fixed to 0.3 ). These results suggest the potential presence of an emission line; however our results are not conclusive.

In Figure 9 we plot the fractional rms amplitude vs. the flux of the disc component (left panel) and the fractional rms amplitude vs. the flux of the Comptonised component (right panel). In the left panel of Figure 9, as the disc flux increases, the fractional rms amplitude initially remains con- 

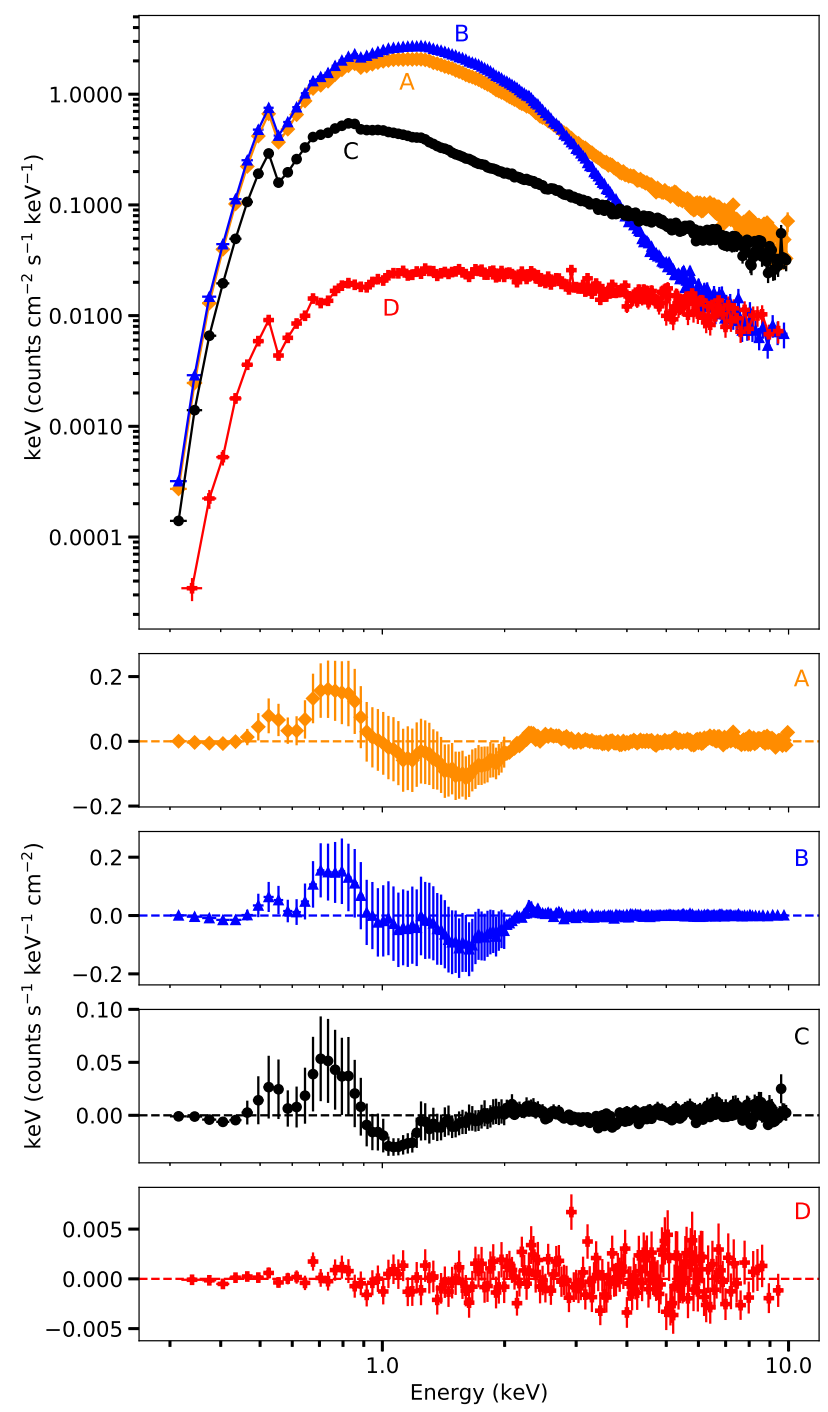

Figure 7. Upper panel: Representative energy spectra corresponding to the different phases of the outburst. Colours and symbols represent the different phases as described in Figure 1. Dashed lines represent the best fit model in each case. Panel A, $\mathrm{B}, \mathrm{C}$ and D: Residuals of the energy spectra corresponding to observations of phase $\mathrm{A}$, phase $\mathrm{B}$, phase $\mathrm{C}$ and phase $\mathrm{D}$ of the outburst, respectively.

stant, phase D, and then decreases as the disc flux increases further, C, A and B. While during phase B the rms amplitude is consistent with being constant, those measurements are consistent with the overall trend of the rms amplitude with disc flux, and extend the anti-correlation shown by the measurements in phases $\mathrm{C}$ and $\mathrm{A}$. On the contrary, when we plot the rms amplitude vs. the Comptonised flux (right panel of Figure 9), the relation is not continuous since in those cases phase B is in between phase D and C (see Figure 8).

\section{DISCUSSION}

In this paper we present a detailed spectral and timing study of the 2018 outburst of MAXI J1727-203. We found that the system showed three different spectral states during this outburst. Fitting the energy spectra of the source with a combination of a soft thermal component and a hard Comptonised component, we found that the photon index ranges between $\sim 1.75$ and $\sim 3.1$ and the temperature at the inner disc radius ranged between $0.1 \mathrm{keV}$ and $0.45 \mathrm{keV}$. From MJD 58342 until the end of the outburst, the disc component is not detected. The power spectra of MAXI J1727-203 showed broadband noise up to $\sim 20 \mathrm{~Hz}$, without any significant QPOs. The 0.01-64 Hz averaged fractional rms amplitude $(0.5-12 \mathrm{keV})$ ranged from $<1 \%$ to $\sim 30 \%$. In addition, we found that the fractional rms amplitude increased with energy during most of the outburst, except at the end of the outburst when it remained approximately constant with energy. All these properties allow us to discuss the nature of the compact object of MAXI J1727-203 and to determine the spectral states that characterise the source during the outburst.

Before comparing our results with previous works, it is important to note that NICER observations are sensitive in the $0.5-12 \mathrm{keV}$ range, whereas most of our understanding of LMXBs in the last two decades comes from observations done with the Principal Counter Array (PCA) in Rossi Xray Timing Explorer (RXTE; Bradt et al. 1993), which was more sensitive in the $3-25 \mathrm{keV}$ range. NICER observations, therefore, will be more affected by the interstellar absorption (which affects mainly the spectrum $<3 \mathrm{keV}$ ) than those of RXTE, affecting not only the energy spectra but also the colours/hardness estimated from them. Especially important is as well the role of the disc component of the spectra on the amplitude of the variability we detect (Uttley et al. 2011). This is especially important for QPOs, but can also affect the broadband noise. So, for example, the integrated rms amplitudes we report in the previous section are likely underestimated as compared to those we would have measured in the usual RXTE $3-25 \mathrm{keV}$ energy band-pass. For this work, the difference in energy range probably had an impact on the q-shape loops in the HIDs and RID, as well as the correlations seen in the HRD. In the comparisons below, we at first neglect the energy range difference as we compare our results with those based on $R X T E$ data but then compare with recent results based on NICER observations. Our conclusions are not affected by the difference in the energy range used.

\subsection{Nature of the compact object in MAXI J1727-203}

The nature of the compact object in MAXI J1727-203 is still under debate. Negoro et al. (2018) suggested that the source is a BH LMXB. However, the dynamical mass of the system has not been estimated yet, and the lack of very clear NS signatures (i.e. X-ray pulsations and thermonuclear X-ray bursts) does not allow to determine with absolute certainty the nature of the compact object. Below we use the evolution of the spectral and timing properties to investigate whether the compact object is a $\mathrm{BH}$ or a NS.

The track traced by MAXI J1727-203 in the HID ap- 

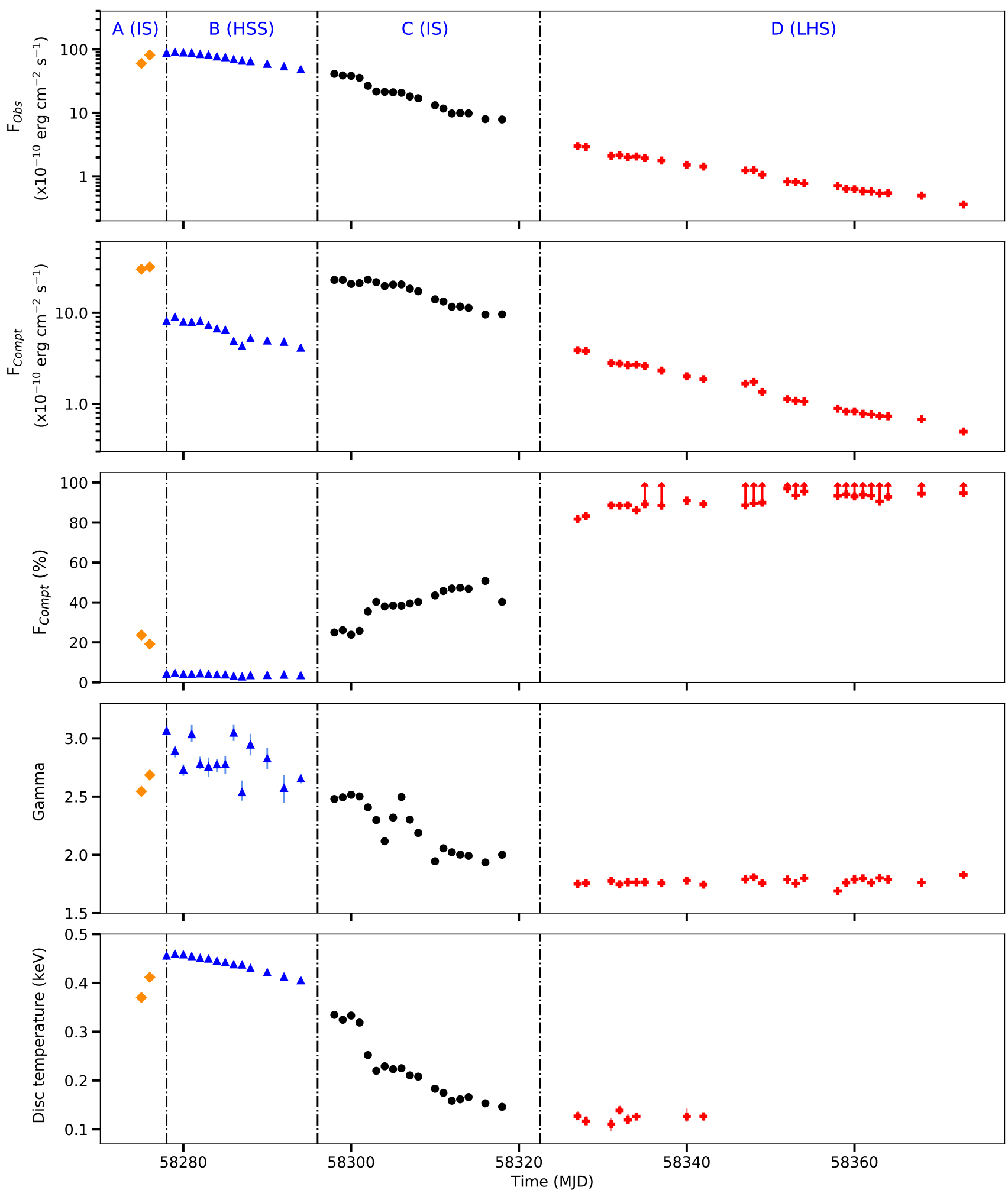

Figure 8. Evolution of the spectral parameters of MAXI J1727-203 in the $0.3-10 \mathrm{keV}$ energy band. We fit NICER X-ray spectra using a TBABS $\times$ (NTHCOMP+DiskBB) model. From top to bottom, we plot the total observed flux, the unabsorbed Comptonised flux $\left(F_{C o m p t}\right)$, the contribution of the Comptonised component to the total unabsorbed flux $\left(F_{C o m p t}(\%)\right)$, the photon index of NTHCOMP and the temperature at the inner disc radius of DISkBB. After MJD 58342 the DISKBB component is not statistically required. The values of $F_{\text {Compt }}(\%)$ after MJD 58342 are not $100 \%$ as those take into account the contribution to the flux of a disc (at 95\% upper limits). The red arrows on the third panel represent the lower limits of the $F_{C o m p t}(\%)$ in observations where the disc was not significantly detected. The different colours represent the different phases of the outburst as defined in the previous Figures. The dashed line points separate the different phases. 

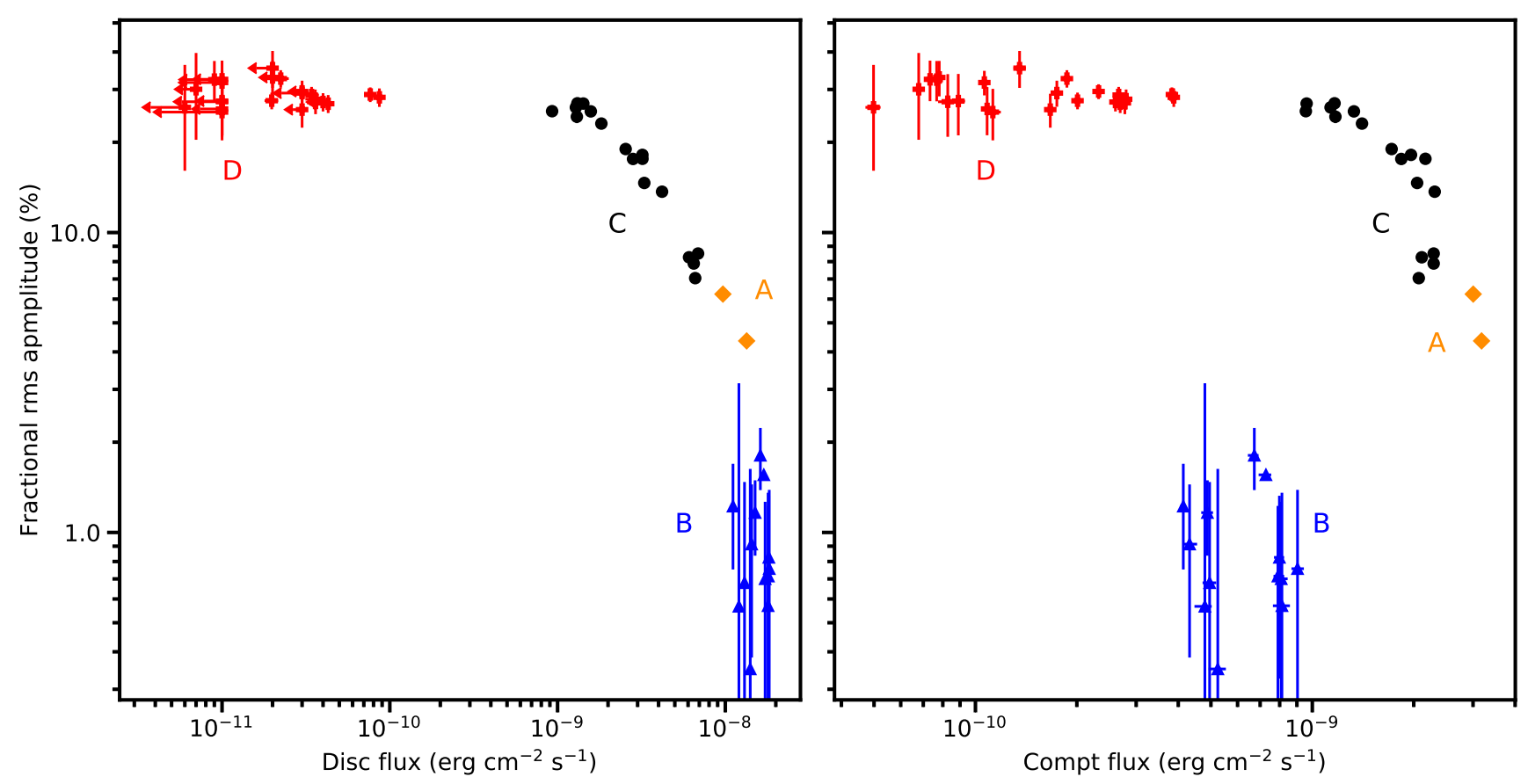

Figure 9. Plot of the $0.01-64 \mathrm{~Hz}$ fractional rms amplitude $(0.5-12 \mathrm{keV})$ versus the flux of the disc component for MAXI J1727-203. Colours and symbols represents the different phases of the outburst as in the previous plots. Red arrows represent the $95 \%$ upper limits of the disc flux.

pears to trace part of the q-track, although we missed the rising part of the outburst. This hysteresis loop is typical of BH LMXBs (e.g. Homan \& Belloni 2005; Remillard \& McClintock 2006; Fender et al. 2009, and references therein). However, hysteresis loops have also been observed in NS LMXBs (Körding et al. 2008; Muñoz-Darias et al. 2014).

An evolution in the HRD similar to that of MAXI J1727-203 has been observed in other LMXBs. The track we found for MAXI J1727-203 is similar to the one showed by GX 339-4 (Belloni et al. 2005) and MAXI J1348630 (Zhang et al. in prep.), both BH LMXBs. Nevertheless, similar tracks were observed in two NS LMXBs (Aql X-1 and 4U 1705-44, Muñoz-Darias et al. 2014).

The track that MAXI J1727-203 traced in the RID is similar to the track traced by the data of the $\mathrm{BH}$ candidate GX 339-4 (based on RXTE; Muñoz-Darias et al. 2011) and MAXI J1348-630 (based on NICER data; Zhang et al. in prep). The difference between MAXI J1727-203 and GX 339-4 is that the Adjacent Hard Line of the latter was located between $30 \%$ and $40 \%$ fractional rms amplitude, whereas in the case of MAXI J1727-203 the Adjacent Hard Line line was located between $20-30 \%$ fractional rms. Muñoz-Darias et al. (2014) found that NS LMXBs also show hysteresis in the RID. In particular, these authors found that low accretion rate NS LMXBs traced similar tracks in the RID as those traced by BH LMXBs. The main difference between these low accretion rate NS systems and $\mathrm{BH}$ systems is that the track followed during the state transitions is diagonal in NS, while state transitions in BH are usually horizontal, at least for low-inclination systems (Muñoz-Darias et al. 2013). This makes low accretion rate NS brighter during the soft than during the hard or intermediate states in the $3-15 \mathrm{keV}$ energy band, as opposed to low-inclination BH LMXBs (Muñoz-Darias et al. 2013). In Figures 2 and 3, it is observed that, unlike the transitions found by MuñozDarias et al. (2014), the transition from the right to the left part of the diagrams are horizontal, suggesting a $\mathrm{BH}$ nature for MAXI J1727-203.

The X-ray timing properties of MAXI J1727-203 do not allow us to determine the nature of the compact object in the system due to the lack of specific $\mathrm{BH}$ and NS signatures. The presence of $\mathrm{kHz}$ QPOs or X-ray pulsations and type-A, $\mathrm{B}$ and $\mathrm{C}$ QPOs would have allowed us to identify the compact object as a NS or a $\mathrm{BH}$, respectively. Unfortunately, no $\mathrm{kHz}$ QPOs or X-ray pulsations are observed in the PDS of MAXI J1727-203. We found some marginally significant QPOs in the PDS at low frequencies (from 0.2 to $6 \mathrm{~Hz}$ ); however the data are not sufficient to identify them with the NS or BH QPO counterparts. We can focus on the maximum frequency of the variability of the broadband noise component. MAXI J1727-203 showed broadband noise component that extends up to $20 \mathrm{~Hz}$. Based on the results of Sunyaev \& Revnivtsev (2000), this behaviour is more typical of BHs, since the power spectra of BH LMXBs displays a strong decline at frequencies higher than $10-50 \mathrm{~Hz}$, with no significant variability above $100-200 \mathrm{~Hz}$ (e.g. GX 339-4, GS 1354-644, XTE J1748-288, and 4U 1630-47). NS LMXBs, on the other hand, can show significant variability in the power spectra up to 500-1000 Hz (e.g. 4U1608-522, SAX J1808.4-3658, and $4 \mathrm{U} 0614+091)$. The fact that MAXI J1727-203 showed variability only up to $20 \mathrm{~Hz}$ suggests that the compact object in MAXI J1727-203 is a BH.

The evolution of the energy dependence of the fractional rms amplitude of the broadband noise component at energies 
$0.3-12 \mathrm{keV}$ is consistent with what has been seen in other BH LMXBs in energies above 2-3 keV (e.g. XTE J1550-564 and XTE J1650-50; Gierliński \& Zdziarski 2005). We found that during most of the outburst, the fractional rms amplitude increased with energy. The only exception is shown in the panel (f) of Figure 6 where the rms remained approximately constant with energy. This panel corresponds to the spectrally hardest observations in Figures 2 and 3 (red crosses of the diagrams). Gierliński \& Zdziarski (2005) found that in the hard state of XTE J1550-564 and XTE J165050 the rms-spectra remained constant or slightly decreased with energy, while in the intermediate and the soft state the rms spectra increased with energy. Some NS show a similar behaviour (e.g. XTE J1701-462; Bu et al. 2015). The rms spectra of broadband noise components of NS increase with energy in some cases (Bu et al. 2015; Bult 2017, studying XTE J1701-462 and MAXI J0911-655, respectively), as MAXI J1727-203 did during most of the outburst. Unfortunately, neither of those works present the evolution of the rms spectra during a whole outburst, so at the moment we cannot compare the evolution of these sources and that of MAXI J1727-203.

The evolution of the spectral parameters of MAXI J1727-203 is similar to what has been observed in other BH LMXBs and NS LMXBs. The photon index of MAXI J1727-203 ranged from $\sim 1.75$ to $\sim 3.1$. This evolution is similar to two of the most studied BH LMXBs, Cyg X-1 (photon index from $\sim 1.5$ to $\sim 2.7$, Titarchuk 1994) and GX 339-4 (photon index from $\sim 1.5$ to $\sim 2.9$, Plant et al. 2014). We also compared the photon index of MAXI J1727-203 with the photon index of two NS LMXBs: $4 \mathrm{U}$ 1636-53 and MXB 1658-298 during its 2015-2017 outburst. In the case of $4 \mathrm{U} 1636-53$, the photon index ranged from 1.3 to 2.5 (Zhang et al. 2017). This range is similar to the photon index range of MAXI J1727-203. In the case of MXB 1658-298, the photon index ranged from $\sim 1.7$ to $\sim 2.4$ (Sharma et al. 2018). Although the photon index in NS LMXBs might show lower values than for BH LMXBs (something that would have to be tested studying a much larger sample), this potential difference would argue that MAXI J1727-203 is a BH candidate. In terms of the inner disc temperature, MAXI J1727-203 showed a lower temperature than other BH LMXBs. The disc temperature of GX 339-4 ranged from $\sim 0.6$ to $\sim 0.9 \mathrm{keV}$ (Plant et al. 2014) and the temperature of $\mathrm{Cyg} X-1$ ranged between $0.5 \mathrm{keV}$ and $0.6 \mathrm{keV}$ (Shaposhnikov \& Titarchuk 2006). The temperature of $4 \mathrm{U} 1636-53$ ranged from $\sim 0.3$ to $\sim 0.8$ $\mathrm{keV}$ in the best-fitting results (Zhang et al. 2017) and the disc temperature of MXB 1658-298 ranged from $\sim 0.6$ to $\sim 0.9 \mathrm{keV}$ (Sharma et al. 2018). The disc temperature of MAXI J1727-203 was lower than these 4 systems.

A potential explanation for a lower temperature in MAXI J1727-203 than in other sources could be related to the mass of the compact object. Assuming that the accretion disc is at the innermost stable circular orbit (ISCO), the temperature at the inner disc radius is proportional to $\left(\dot{M} / M^{2}\right)^{1 / 4}$, where $\dot{M}$ is the mass accretion rate and $M$ is the mass of the compact object (Frank et al. 2002). According to Muñoz-Darias et al. (2008), $M<6 \mathrm{M}_{\odot}$ for GX 3394. We take the temperature of GX 339-4 in the soft state (0.79 keV) from Plant et al. (2014), and we also take the disc temperature of the softest observation in the HID of MAXI J1727-203 (0.45 keV). From that, if we assume that $\dot{M}$ is the same for two sources in the same spectral state, we estimate a lower limit for the mass of MAXI J1727-203 of $\sim 19 \mathrm{M}_{\odot}$. Therefore, the high mass of the compact object can explain the low temperature of the inner disc. Alternatively, as suggested by Gou et al. (2011), the low temperature at the inner disc radius can be a consequence of a low inclination of the accretion disc with respect to the line of sight.

We can repeat this analysis to estimate the mass of MAXI J1727-203 with the NS Aql X-1. For this, we took the temperature of Aql X-1 in the soft state of its 2007 outburst $(\sim 0.66 \mathrm{keV}$, Raichur et al. 2011). Considering a mass of $\sim$ $1.4 \mathrm{M}_{\odot}$ for the NS in Aql X-1, we obtained a mass of $\sim 2 \mathrm{M}_{\odot}$ for the compact object in MAXI J1727-203. If we consider a higher mass for Aql X-1, the mass of MAXI J1727-203 also increases. Considering the latter, this mass estimates suggest that the compact object in MAXI J1727-203 is massive NS or a low-mass BH.

Based on all the above comparisons, although we cannot unambiguously identify the nature of the compact object in MAXI J1727-203, the evolution in the HID, RID and RHD, and the temperature at the inner radius of the accretion disc during the softest observations, suggest that it is a $\mathrm{BH}$.

\subsection{Anticorrelation between the fractional rms amplitude and the flux of the disc component}

Figure 9 shows that the relation between the fractional rms amplitude and the disc flux is continuous and that both quantities are anti-correlated during phases $\mathrm{A}$ and $\mathrm{C}$ (orange diamonds and black circles) of the outburst. While in phases $\mathrm{B}$ and $\mathrm{D}$ (blue triangles and red crosses) the rms amplitude is consistent with being independent of the disc flux, those measurements extend the relation seen in phases $\mathrm{A}$ and $\mathrm{C}$ to low (phase D) and high (phase B) values of the disc flux.

The simplest interpretation of this behaviour is that the variability is produced by the Comptonised component, the disc emission is not variable and, as the relative contribution of the disc to the total emission increases, the variability decreases. If this is the case, the intrinsic variability would be produced by the corona (e.g., for QPOs Lee \& Miller 1998; Lee et al. 2001; Kumar \& Misra 2014). Karpouzas et al. (2020) explain this for the kilohertz QPOs in neutronstar LMXBs, and Zhang et al. (2020) for the type C QPOs in the black-hole candidate GRS $1915+105$, but the same mechanism could apply for the broadband component that we discuss here, but the observed trend would be driven by the disc. A similar argument was discussed by Méndez et al. (2001) for the dependence of the rms amplitude of the kilohertz QPOs in the neutron-star LMXBs 4U 1728-34, $4 \mathrm{U} 1608-52$ and Aql X-1.

\subsection{Spectral states of MAXI J1727-203}

Assuming that the source is a BH LMXB, we can identify its spectral states from its spectral and timing properties. Here we describe the different spectral states found for MAXI J1727-203:

- Low/hard state (LHS): From MJD 58327 to MJD 
58397. This period corresponds with phase D of the outburst, marked with red filled crosses in Figures 1, 2, 3, 8 and 9. In the HID the source was in the right vertical branch, with hardness values close to $\sim 0.1$. The fractional rms amplitude in the LHS state was close to $\sim 30 \%$ and followed the AHL in the RID, supporting the LHS classification state based on the results of Muñoz-Darias et al. (2011). The fractional rms amplitude was also approximately constant with energy (panel with red filled crosses in Figure 6). The PDS was dominated by a broadband noise component (panel (iii) in Figure 4). In terms of spectral properties, the contribution of the Comptonised component was $>80 \%$ in this state. At the end of the outburst, the disc component is not significant. The fractional rms amplitude is not correlated with the flux of the Comptonised component. This is because the fractional $\mathrm{rms}$ remains approximately constant with energy. The photon index of the Comptonised component and the inner disc temperature remained approximately constant at $\sim 1.8$ and $\sim 0.1 \mathrm{keV}$, respectively.

- Intermediate states (IS): From MJD 58274 to MJD 58278 and from MJD 58298 to MJD 58327. These periods corresponds to phases $\mathrm{A}$ and $\mathrm{C}$ of the outburst, respectively, which are marked with orange diamonds (phase A) and black circles (phase C) in Figures 1, 2, 3, 8 and 9. In the HID these correspond to the horizontal branches with hardness values from $\sim 0.02$ to $\sim 0.1$. In these periods, the fractional rms amplitude ranged from $\sim 5 \%$ to $\sim 30 \%$ and the source evolved to the top left part of the RID in the first epoch (MJD 58274-MJD 58278) and to the Adjacent Hard Line in the second epoch (MJD 58298-MJD 58327), as can be seen in Figure 3. The fractional rms amplitude increased with energy (panels with black circles and orange diamonds in Figure 6), and the PDS was dominated by a broadband noise component (panel (ii) in Figure 4). No significant QPOs are detected during this phase. The characteristic frequency also increases with the intensity. The contribution of the Comptonised component ranged from $\sim 20 \%$ to $50 \%$. The fractional rms amplitude and the flux of the Comptonised flux are anticorrelated, suggesting that the change of variability is driven by changes in the flux of the Comptonised component. The photon index of the Comptonised component in this state ranged from $\sim 2.0$ to $\sim 2.7$ and the inner disc temperature decreased from $\sim 0.4 \mathrm{keV}$ to $\sim 0.15 \mathrm{keV}$.

- High/soft state (HSS): From MJD 58278 to MJD 58298. This period corresponds to phase B of the outburst, plotted with blue triangles in Figures 1, 2, 3, 8 and 9. In the HID the hardness ratio was approximately constant close to $\sim 0.005$. The fractional $\mathrm{rms}$ amplitude was $\sim 1 \%$, which can be observed in the top left part of the RID, where the source evolved around the $1 \%$ fractional $\mathrm{rms}$ line. The maximum frequency also increases with energy. The rms spectrum increases with energy (blue triangles in Figure 6). The contribution of the Comptonised component was less than $5 \%$, the photon index of the Comptonised component ranged from $\sim 2.5$ to $\sim 3.0$ and the temperature of the inner disc decreased from $\sim 0.45 \mathrm{keV}$ to $\sim 0.3 \mathrm{keV}$. The rms-flux correlation was flat with some scatter (Figure 9).

\section{ACKNOWLEDGEMENTS}

This work is based on observations made by the NICER Xray mission supported by NASA. This research has made use of data and software provided by the High Energy Astrophysics Science Archive Research Center (HEASARC), a service of the Astrophysics Science Division at NASA/GSFC and the High Energy Astrophysics Division of the Smithsonian Astrophysical Observatory. This research has made use of the MAXI light curve provided by RIKEN, JAXA, and the MAXI team. This research has also made use of Swift/BAT transient monitor results provided by the Swift/BAT team. KA acknowledges support from a UGCUKIERI Phase 3 Thematic Partnership (UGC-UKIERI2017-18-006; PI: P. Gandhi). KA especially acknowledges Dr. Keith Arnaud for his help with the X-ray tool XSPEC. D.A. and D.J.K.B. acknowledge support from the Royal Society. V.A.C. acknowledges support from the Royal Society International Exchanges "The first step for High-Energy Astrophysics relations between Argentina and UK" and from the Spanish Ministerio de Ciencia e Innovación under grant AYA2017-83216-P. LZ and AC acknowledges support from the Royal Society Newton International Fellowship. R.M.L. acknowledges the support of NASA through Hubble Fellowship Program grant HST-HF2-51440.001.

\section{DATA AVAILABILITY}

The data underlying this article are publicly available in the High Energy Astrophysics Science Archive Research Center (HEASARC) at

The data underlying this article are publicly available in the High Energy Astrophysics Science Archive Research Center (HEASARC) at https://heasarc.gsfc.nasa.gov/ $\mathrm{db}$-perl/W3Browse/w3browse.pl

\section{REFERENCES}

Arnaud K. A., 1996, in Jacoby G. H., Barnes J., eds, Astronomical Society of the Pacific Conference Series Vol. 101, Astronomical Data Analysis Software and Systems V. p. 17

Belloni T. M., 2010, in Belloni T., ed., Vol. 794, The Jet Paradigm, Lecture Notes in Physics. ISBN 978-3-540-76936-1. Springer-Verlag Berlin Heidelberg. p. 53, doi:10.1007/978-3540-76937-8_3

Belloni T. M., Stella L., 2014, Space Sci. Rev., 183, 43

Belloni T., Psaltis D., van der Klis M., 2002, ApJ, 572, 392

Belloni T., Homan J., Casella P., van der Klis M., Nespoli E., Lewin W. H. G., Miller J. M., Méndez M., 2005, A\&A, 440, 207

Belloni T., et al., 2006, MNRAS, 367, 1113

Belloni T. M., Motta S. E., Muñoz-Darias T., 2011, Bulletin of the Astronomical Society of India, 39, 409

Bradt H. V., Rothschild R. E., Swank J. H., 1993, A\&AS, 97, 355

Bu Q.-c., Chen L., Li Z.-s., Qu J.-l., Belloni T. M., Zhang L., 2015, ApJ, 799, 2

Bult P., 2017, ApJ, 837, 61

Burke M. J., Gilfanov M., Sunyaev R., 2017, MNRAS, 466, 194

Capitanio F., Belloni T., Del Santo M., Ubertini P., 2009, MNRAS, 398, 1194

Casella P., Belloni T., Homan J., Stella L., 2004, A\&A, 426, 587

Di Salvo T., et al., 2000, ApJ, 544, L119

Done C., Gierliński M., 2003, MNRAS, 342, 1041 
Done C., Gierliński M., Kubota A., 2007, Astronomy and Astrophysics Review, 15, 1

Dunn R. J. H., Fender R. P., Körding E. G., Belloni T., Cabanac C., 2010, MNRAS, 403, 61

Fender R. P., Homan J., Belloni T. M., 2009, MNRAS, 396, 1370

Frank J., King A., Raine D. J., 2002, Accretion Power in Astrophysics: Third Edition. Cambridge, UK: Cambridge University Press

Gao H. Q., et al., 2017, MNRAS, 466, 564

Gehrels N., et al., 2004, ApJ, 611, 1005

Gendreau K. C., Arzoumanian Z., Okajima T., 2012, in Space Telescopes and Instrumentation 2012: Ultraviolet to Gamma Ray. p. 844313, doi:10.1117/12.926396

Gierliński M., Done C., 2002, MNRAS, 337, 1373

Gierliński M., Zdziarski A. A., 2005, MNRAS, 363, 1349

Gou L., et al., 2011, ApJ, 742, 85

Hasinger G., van der Klis M., 1989, A\&A, 225, 79

Homan J., Belloni T., 2005, Ap\&SS, 300, 107

Homan J., Wijnands R., van der Klis M., Belloni T., van Paradijs J., Klein-Wolt M., Fender R., Méndez M., 2001, ApJS, 132, 377

Karpouzas K., Méndez M., Ribeiro E. r. M., Altamirano D., Blaes O., García F., 2020, MNRAS, 492, 1399

Kennea J. A., Bahramian A., Beardmore A. P., 2018, The Astronomer's Telegram, 11697

Klein-Wolt M., van der Klis M., 2008, ApJ, 675, 1407

Körding E., Rupen M., Knigge C., Fender R., Dhawan V., Templeton M., Muxlow T., 2008, Science, 320, 1318

Kumar N., Misra R., 2014, MNRAS, 445, 2818

Leahy D. A., Darbro W., Elsner R. F., Weisskopf M. C., Sutherland P. G., Kahn S., Grindlay J. E., 1983, ApJ, 266, 160

Lee H. C., Miller G. S., 1998, MNRAS, 299, 479

Lee H. C., Misra R., Taam R. E., 2001, ApJ, 549, L229

Lin D., Remillard R. A., Homan J., 2007, ApJ, 667, 1073

Ludlam R. M., et al., 2018a, ApJ, 858, L5

Ludlam R. M., et al., 2018b, The Astronomer's Telegram, 11689

Méndez M., van der Klis M., 1997, ApJ, 479, 926

Méndez M., van der Klis M., Ford E. C., 2001, ApJ, 561, 1016

Miller J. M., et al., 2018, ApJ, 860, L28

Mitsuda K., et al., 1984, PASJ, 36, 741

Miyamoto S., Kitamoto S., Hayashida K., Egoshi W., 1995, ApJ, 442, L13

Motta S. E., 2016, Astronomische Nachrichten, 337, 398

Motta S., Homan J., Muñoz Darias T., Casella P., Belloni T. M., Hiemstra B., Méndez M., 2012, MNRAS, 427, 595

Muñoz-Darias T., Casares J., Martínez-Pais I. G., 2008, MNRAS, 385,2205

Muñoz-Darias T., Motta S., Belloni T. M., 2011, MNRAS, 410, 679

Muñoz-Darias T., Coriat M., Plant D. S., Ponti G., Fender R. P., Dunn R. J. H., 2013, MNRAS, 432, 1330

Muñoz-Darias T., Fender R. P., Motta S. E., Belloni T. M., 2014, MNRAS, 443, 3270

Negoro H., et al., 2018, The Astronomer's Telegram, 11696

Plant D. S., Fender R. P., Ponti G., Muñoz-Darias T., Coriat M., 2014, MNRAS, 442, 1767

Raichur H., Misra R., Dewangan G., 2011, MNRAS, 416, 637

Remillard R. A., McClintock J. E., 2006, Annual Review of Astronomy and Astrophysics, 44, 49

Shakura N. I., Sunyaev R. A., 1973, in Bradt H., Giacconi R., eds, IAU Symposium Vol. 55, X- and Gamma-Ray Astronomy. p. 155

Shaposhnikov N., Titarchuk L., 2006, ApJ, 643, 1098

Sharma R., Jaleel A., Jain C., Pand ey J. C., Paul B., Dutta A., 2018, MNRAS, 481, 5560

Sunyaev R., Revnivtsev M., 2000, A\&A, 358, 617

Sunyaev R. A., Titarchuk L. G., 1980, A\&A, 86, 121

Sunyaev R. A., Truemper J., 1979, Nature, 279, 506
Tanaka Y., 1989, in Hunt J., Battrick B., eds, ESA Special Publication Vol. 1, Two Topics in X-Ray Astronomy, Volume 1: X Ray Binaries. Volume 2: AGN and the X Ray Background. p. 3

Titarchuk L., 1994, ApJ, 434, 570

Tomsick J., Shaw A., Garcia J., Walton D., Fuerst F., Miller J., Parker M., Xu Y., 2018, The Astronomer's Telegram, 11881

Uttley P., Klein-Wolt M., 2015, MNRAS, 451, 475

Uttley P., Wilkinson T., Cassatella P., Wilms J., Pottschmidt K., Hanke M., Böck M., 2011, MNRAS, 414, L60

Verner D. A., Ferland G. J., Korista K. T., Yakovlev D. G., 1996, ApJ, 465, 487

Wang-Ji J., et al., 2018, ApJ, 855, 61

Wijnands R., Homan J., van der Klis M., 1999, ApJ, 526, L33

Wilms J., Allen A., McCray R., 2000, ApJ, 542, 914

Yoneyama T., et al., 2018, The Astronomer's Telegram, 11683

Zdziarski A. A., Gierliński M., 2004, Progress of Theoretical Physics Supplement, 155, 99

Zdziarski A. A., Johnson W. N., Magdziarz P., 1996, MNRAS, 283, 193

Zhang G., Méndez M., Sanna A., Ribeiro E. M., Gelfand J. D., 2017, MNRAS, 465, 5003

Zhang L., et al., 2020, MNRAS,

Życki P. T., Done C., Smith D. A., 1999, MNRAS, 309, 561

van Doesburgh M., van der Klis M., Morsink S. M., 2018, MNRAS, 479,426

van der Klis M., 1989, ARA\&A, 27, 517

van der Klis M., 1994, ApJS, 92, 511

van der Klis M., 1995, in Alpar M. A., Kiziloglu U., van Paradijs J., eds, NATO Advanced Science Institutes (ASI) Series C Vol. 450, NATO Advanced Science Institutes (ASI) Series C. p. 301

van der Klis M., 2000, ARA\&A, 38, 717

van der Klis M., 2006, in In: Compact stellar X-ray sources. Edited by Walter Lewin \&amp; Michiel van der Klis. Cambridge Astrophysics Series, No. 39. Cambridge, UK: Cambridge University Press, ISBN 978-0-521-82659-4, ISBN 0521-82659-4, DOI: $10.2277 / 0521826594,2006$, p. 39 - 112. pp $39-112$

\section{APPENDIX A: SPECTRAL FITTING PARAMETERS}


Table A1. Summary of the spectral parameters of MAXI J1727-203 in the 0.3-10 keV energy band. Errors represent the $1 \sigma$ level confidence interval of the parameter. For the observations in which we do not detect the disc component significantly we give the $95 \%$ confidence upper limit of the disc normalisation and the corresponding disc flux.

\begin{tabular}{|c|c|c|c|c|c|c|c|c|c|}
\hline $\begin{array}{l}\text { MJD } \\
\text { NICER }\end{array}$ & $\Gamma$ & $\begin{array}{l}\text { NTHCOMP norm. } \\
\qquad\left(\times 10^{-2}\right)\end{array}$ & $\begin{array}{l}k T_{\text {in }} \\
(\mathrm{keV})\end{array}$ & $\begin{array}{c}\text { DISKBB norm. } \\
{\left[\times 10^{4} \mathrm{~km}^{2}(10 \mathrm{kpc})^{-2}\right]}\end{array}$ & $\begin{array}{l}\text { Comptonised flux } \\
\left(\times 10^{-10} \mathrm{erg} \mathrm{cm}^{-2} \mathrm{~s}^{-1}\right)\end{array}$ & $\begin{array}{c}\text { Disc flux } \\
\left(\times 10^{-10} \mathrm{erg} \mathrm{cm}^{-2} \mathrm{~s}^{-1}\right)\end{array}$ & $\begin{array}{l}\text { Unabsorbed flux } \\
\left(\times 10^{-10} \mathrm{erg} \mathrm{cm}^{-2} \mathrm{~s}^{-1}\right)\end{array}$ & $\begin{array}{l}\text { Total absorbed flux } \\
\left(\times 10^{-10} \mathrm{erg} \mathrm{cm}^{-2} \mathrm{~s}^{-1}\right)\end{array}$ & $\begin{array}{l}\text { Phase } \\
\text { outburst }\end{array}$ \\
\hline 58275 & $2.53 \pm 0.03$ & $50.9 \pm 0.1$ & $0.370 \pm 0.001$ & $2.99 \pm 0.03$ & $30.0 \pm 0.3$ & $96.7_{-1.0}^{+0.6}$ & $126.8_{-1.0}^{+0.7}$ & $60.2 \pm 0.2$ & A \\
\hline 58276 & $2.68 \pm 0.03$ & $50.5 \pm 0.2$ & $0.412 \pm 0.003$ & $2.62 \pm 0.08$ & $31.8 \pm 0.5$ & $134.0 \pm 0.9$ & $166 \pm 1$ & $81.3_{-0.3}^{+0.4}$ & A \\
\hline 58278 & $3.1 \pm 0.1$ & $12.7 \pm 0.1$ & $0.456 \pm 0.002$ & $2.28 \pm 0.04$ & $8.1 \pm 0.5$ & $179.8 \pm 0.4$ & $187.9 \pm 0.6$ & $88.00_{-0.06}^{+0.09}$ & B \\
\hline 58279 & $2.89 \pm 0.05$ & $13.3 \pm 0.1$ & $0.459 \pm 0.002$ & $2.24 \pm 0.04$ & $9.0 \pm 0.4$ & $183.0 \pm 0.8$ & $192.0 \pm 0.8$ & $90.7 \pm 0.3$ & B \\
\hline 58280 & $2.73 \pm 0.05$ & $11.1 \pm 0.1$ & $0.458 \pm 0.002$ & $2.26 \pm 0.04$ & $8.0 \pm 0.3$ & $181.7 \pm 0.9$ & $189.8 \pm 0.9$ & $89.3 \pm 0.4$ & B \\
\hline 58281 & $3.04 \pm 0.07$ & $12.33 \pm 0.08$ & $0.455 \pm 0.001$ & $2.32 \pm 0.02$ & $7.9 \pm 0.3$ & $181.0 \pm 0.8$ & $188.4 \pm 0.9$ & $88.0 \pm 0.3$ & B \\
\hline 58282 & $2.78 \pm 0.05$ & $11.71 \pm 0.09$ & $0.451 \pm 0.002$ & $2.29 \pm 0.04$ & $8.1 \pm 0.4$ & $173.0 \pm 0.8$ & $181.0 \pm 0.8$ & $84.2 \pm 0.3$ & B \\
\hline 58283 & $2.76 \pm 0.09$ & $10.5 \pm 0.1$ & $0.449 \pm 0.002$ & $2.28 \pm 0.04$ & $7.3 \pm 0.4$ & $169.6 \pm 0.8$ & $176.9 \pm 0.9$ & $81.9 \pm 0.3$ & B \\
\hline 58284 & $2.78_{-0.07}^{+0.04}$ & $9.90 \pm 0.09$ & $0.445 \pm 0.001$ & $2.27 \pm 0.02$ & $6.7 \pm 0.3$ & $161.8 \pm 0.8$ & $169.0 \pm 0.9$ & $77.3 \pm 0.3$ & B \\
\hline 58285 & $2.78 \pm 0.08$ & $9.67 \pm 0.07$ & $0.442 \pm 0.001$ & $2.28 \pm 0.02$ & $6.5 \pm 0.3$ & $158.3 \pm 0.4$ & $164.8 \pm 0.5$ & $75.1 \pm 0.2$ & B \\
\hline 58286 & $3.05 \pm 0.07$ & $8.06 \pm 0.07$ & $0.438 \pm 0.001$ & $2.27 \pm 0.02$ & $4.9 \pm 0.2$ & $150.5 \pm 0.7$ & $155.4 \pm 0.7$ & $69.8 \pm 0.2$ & B \\
\hline 58287 & $2.54 \pm 0.08$ & $5.90 \pm 0.06$ & $0.437 \pm 0.001$ & $2.17 \pm 0.02$ & $4.3 \pm 0.2$ & $143.5 \pm 0.5$ & $147.8 \pm 0.6$ & $66.3 \pm 0.1$ & B \\
\hline 58288 & $2.95 \pm 0.09$ & $8.57 \pm 0.09$ & $0.430 \pm 0.001$ & $2.28 \pm 0.02$ & $5.2 \pm 0.3$ & $140.8 \pm 0.7$ & $146.0 \pm 0.7$ & $64.9 \pm 0.2$ & B \\
\hline 58290 & $2.83 \pm 0.09$ & $8.00 \pm 0.08$ & $0.421 \pm 0.002$ & $2.30 \pm 0.04$ & $5.0 \pm 0.2$ & $130.2 \pm 0.7$ & $135.1 \pm 0.8$ & $59.1 \pm 0.3$ & B \\
\hline 58292 & $2.6 \pm 0.1$ & $7.2 \pm 0.1$ & $0.413 \pm 0.002$ & $2.34 \pm 0.05$ & $4.8 \pm 0.3$ & $121 \pm 1$ & $125 \pm 1$ & $53.9 \pm 0.3$ & B \\
\hline 58294 & $2.66 \pm 0.04$ & $6.55 \pm 0.05$ & $0.4053 \pm 0.0007$ & $2.32 \pm 0.02$ & $4.14 \pm 0.08$ & $111.0 \pm 0.6$ & $115.2 \pm 0.6$ & $48.7 \pm 0.2$ & B \\
\hline 58298 & $2.48 \pm 0.02$ & $42.1 \pm 0.1$ & $0.330 \pm 0.001$ & $3.29 \pm 0.04$ & $22.9 \pm 0.2$ & $68.8 \pm 0.6$ & $91.7 \pm 0.6$ & $41.1 \pm 0.1$ & $\mathrm{C}$ \\
\hline 58299 & $2.49 \pm 0.02$ & $43.7 \pm 0.1$ & $0.324 \pm 0.002$ & $3.55 \pm 0.09$ & $22.9 \pm 0.3$ & $64.8 \pm 0.5$ & $87.8 \pm 0.6$ & $38.8 \pm 0.1$ & C \\
\hline 58300 & $2.52 \pm 0.02$ & $38.9 \pm 0.1$ & $0.333 \pm 0.002$ & $3.23 \pm 0.08$ & $20.7 \pm 0.2$ & $66.2 \pm 0.9$ & $86.9 \pm 0.9$ & $38.2 \pm 0.2$ & C \\
\hline 58301 & $2.50 \pm 0.02$ & $41.1 \pm 0.1$ & $0.319 \pm 0.002$ & $3.59 \pm 0.09$ & $21.1 \pm 0.3$ & $60.8 \pm 0.4$ & $81.9 \pm 0.5$ & $35.60_{-0.03}^{+0.08}$ & $\mathrm{C}$ \\
\hline 58302 & $2.41 \pm 0.02$ & $51.2 \pm 0.02$ & $0.252 \pm 0.003$ & $7.1 \pm 0.3$ & $23.1 \pm 0.3$ & $41.9 \pm 0.6$ & $65.1 \pm 0.6$ & $26.7 \pm 0.1$ & $\mathrm{C}$ \\
\hline 58303 & $2.30 \pm 0.01$ & $48.3 \pm 0.1$ & $0.220 \pm 0.003$ & $10.1 \pm 0.6$ & $21.7 \pm 0.2$ & $32.1 \pm 0.5$ & $53.7 \pm 0.5$ & $21.70 \pm 0.09$ & $\mathrm{C}$ \\
\hline 58304 & $2.12 \pm 0.01$ & $37.9 \pm 0.1$ & $0.229 \pm 0.002$ & $8.3 \pm 0.3$ & $19.6 \pm 0.2$ & $32.0 \pm 0.4$ & $51.7 \pm 0.4$ & $21.40 \pm_{-0.09}^{+0.05}$ & C \\
\hline 58305 & $2.32 \pm 0.02$ & $45.7 \pm 0.2$ & $0.223 \pm 0.003$ & $9.6 \pm 0.5$ & $20.4 \pm 0.3$ & $32.7 \pm 0.6$ & $53.1 \pm 0.6$ & $21.1 \pm 0.1$ & $\mathrm{C}$ \\
\hline 58306 & $2.50 \pm 0.02$ & $50.6 \pm 0.3$ & $0.225 \pm 0.004$ & $9.3 \pm 0.6$ & $20.5 \pm 0.3$ & $32.9 \pm 0.4$ & $53.4 \pm 0.6$ & $20.70 \pm 0.06$ & $\mathrm{C}$ \\
\hline 58307 & $2.30 \pm 0.02$ & $41.7 \pm 0.2$ & $0.210 \pm 0.003$ & $10.9 \pm 0.6$ & $18.4 \pm 0.3$ & $28.2 \pm 0.4$ & $46.6 \pm 0.5$ & $18.10 \pm 0.08$ & $\mathrm{C}$ \\
\hline 58308 & $2.188 \pm 0.009$ & $36.4 \pm 0.1$ & $0.2079 \pm 0.0009$ & $10.4 \pm 0.2$ & $17.20 \pm 0.08$ & $25.5 \pm 0.2$ & $42.7 \pm 0.3$ & $17.00 \pm 0.05$ & C \\
\hline 58310 & $1.944 \pm 0.008$ & $25.5 \pm 0.1$ & $0.183 \pm 0.002$ & $13.7 \pm 0.6$ & $14.04 \pm 0.07$ & $18.2 \pm 0.2$ & $32.3 \pm 0.2$ & $13.2 \pm 0.06$ & C \\
\hline 58311 & $2.05 \pm 0.01$ & $26.5 \pm 0.1$ & $0.175 \pm 0.002$ & $14.9 \pm 0.7$ & $13.29 \pm 0.09$ & $15.8 \pm 0.3$ & $29.1 \pm 0.3$ & $11.70 \pm 0.03$ & $\mathrm{C}$ \\
\hline 58312 & $2.02 \pm 0.02$ & $22.8 \pm 0.2$ & $0.159 \pm 0.002$ & $20 \pm 1$ & $11.64 \pm 0.07$ & $13.1 \pm 0.2$ & $24.8 \pm 0.2$ & $9.79 \pm 0.08$ & C \\
\hline 58313 & $2.00 \pm 0.02$ & $22.6 \pm 0.2$ & $0.161 \pm 0.002$ & $18.0 \pm 0.9$ & $11.7 \pm 0.1$ & $13.0 \pm 0.2$ & $24.7 \pm 0.2$ & $9.96 \pm 0.06$ & $\mathrm{C}$ \\
\hline 58314 & $1.99 \pm 0.01$ & $21.64 \pm 0.08$ & $0.166 \pm 0.002$ & $15.5 \pm 0.8$ & $11.33 \pm 0.06$ & $12.9 \pm 0.3$ & $24.2 \pm 0.3$ & $9.82 \pm 0.04$ & $\mathrm{C}$ \\
\hline 58316 & $1.935_{-0.011}^{+0.008}$ & $17.53 \pm 0.08$ & $0.153 \pm 0.003$ & $17 \pm 1$ & $9.57 \pm 0.07$ & $9.3 \pm 0.3$ & $18.9 \pm 0.3$ & $7.97 \pm 0.04$ & C \\
\hline 58318 & $2.001 \pm 0.009$ & $17.50 \pm 0.09$ & $0.146 \pm 0.004$ & $16 \pm 2$ & $9.6 \pm 0.1$ & $14 \pm 1$ & $24 \pm 1$ & $7.87 \pm 0.04$ & C \\
\hline 58327 & $1.75 \pm 0.03$ & $6.03 \pm 0.05$ & $0.127 \pm 0.008$ & $4 \pm 1$ & $3.88 \pm 0.05$ & $0.87 \pm 0.07$ & $4.75 \pm 0.08$ & $3.00 \pm 0.04$ & D \\
\hline 58328 & $1.76 \pm 0.01$ & $5.97 \pm 0.04$ & $0.117 \pm 0.009$ & $6 \pm 2$ & $3.83 \pm 0.04$ & $0.77 \pm 0.04$ & $4.59 \pm 0.06$ & $2.92 \pm 0.03$ & D \\
\hline 58331 & $1.77 \pm 0.02$ & $4.42 \pm 0.04$ & $0.11 \pm 0.01$ & $4 \pm 1$ & $2.80 \pm 0.06$ & $0.36 \pm 0.05$ & $3.16 \pm 0.07$ & $2.10 \pm 0.02$ & D \\
\hline 58332 & $1.75 \pm 0.01$ & $4.31 \pm 0.03$ & $0.139_{-0.006}^{+0.009}$ & $1.1 \pm 0.3$ & $2.78 \pm 0.03$ & $0.36 \pm 0.04$ & $3.14 \pm 0.05$ & $2.16 \pm 0.02$ & D \\
\hline 58333 & $1.77 \pm 0.01$ & $4.52 \pm 0.03$ & $0.12 \pm 0.09$ & $2.3_{-0.7}^{+1.0}$ & $2.66 \pm 0.03$ & $0.34 \pm 0.04$ & $3.00 \pm 0.04$ & $2.020_{-0.010}^{+0.006}$ & D \\
\hline 58334 & $1.77 \pm 0.02$ & $4.24 \pm 0.03$ & $0.126 \pm 0.007$ & $2.1 \pm 0.5$ & $2.69 \pm 0.03$ & $0.43 \pm 0.03$ & $3.12 \pm 0.04$ & $2.06 \pm 0.02$ & D \\
\hline 58335 & $1.77 \pm 0.01$ & $4.08 \pm 0.02$ & - & $<5$ & $2.60 \pm 0.03$ & $<0.4$ & $2.92 \pm 0.05$ & $1.95 \pm 0.02$ & $\mathrm{D}$ \\
\hline 58337 & $1.76 \pm 0.02$ & $3.63 \pm 0.02$ & - & $<3$ & $2.32 \pm 0.05$ & $<0.3$ & $2.62 \pm 0.05$ & $1.780_{-0.007}^{+0.020}$ & D \\
\hline 58340 & $1.779_{-0.005}^{+0.011}$ & $3.41 \pm 0.02$ & $0.13 \pm 0.02$ & $0.9 \pm 0.3$ & $2.01 \pm 0.03$ & $0.19 \pm 0.02$ & $2.20 \pm 0.04$ & $1.520 \pm 0.006$ & D \\
\hline 58342 & $1.74 \pm 0.02$ & $2.89 \pm 0.02$ & $0.126 \pm 0.009$ & $1.1 \pm 0.3$ & $1.87 \pm 0.03$ & $0.22 \pm 0.03$ & $2.09 \pm 0.04$ & $1.43 \pm 0.01$ & D \\
\hline 58347 & $1.79 \pm 0.02$ & $2.79 \pm 0.03$ & - & $<5$ & $1.67 \pm 0.04$ & $<0.3$ & $1.88 \pm 0.06$ & $1.24 \pm 0.02$ & D \\
\hline 58348 & $1.808_{-0.012}^{+0.007}$ & $2.79 \pm 0.02$ & - & $<19$ & $1.74 \pm 0.04$ & $<0.3$ & $1.95 \pm 0.09$ & $1.260_{-0.007}^{+0.010}$ & D \\
\hline 58349 & $1.76 \pm 0.03$ & $2.09 \pm 0.03$ & - & $<0.6$ & $1.35 \pm 0.06$ & $<0.2$ & $1.50 \pm 0.07$ & $1.06 \pm 0.03$ & D \\
\hline
\end{tabular}




\section{K. Alabarta et al.}

\section{Table A1. Continued}

\begin{tabular}{|c|c|c|c|c|c|c|c|c|c|}
\hline MJD & $\Gamma$ & $\begin{array}{l}\text { NTHCOMP norm. } \\
\qquad\left(\times 10^{-2}\right)\end{array}$ & $\begin{array}{l}k T_{i n} \\
(\mathrm{keV})\end{array}$ & $\begin{array}{c}\text { DISKBB norm. } \\
{\left[\times 10^{4} \mathrm{~km}^{2}(10 \mathrm{kpc})^{-2}\right]}\end{array}$ & $\begin{array}{c}\text { Compt. flux } \\
\left(\times 10^{-10} \mathrm{erg} \mathrm{cm}^{-2} \mathrm{~s}^{-1}\right)\end{array}$ & $\begin{array}{c}\text { Disc flux } \\
\left(\times 10^{-10} \mathrm{erg} \mathrm{cm}^{-2} \mathrm{~s}^{-1}\right)\end{array}$ & $\begin{array}{l}\text { Unabsorbed flux } \\
\left(\times 10^{-10} \mathrm{erg} \mathrm{cm}^{-2} \mathrm{~s}^{-1}\right)\end{array}$ & $\begin{array}{l}\text { Total absorbed flux } \\
\left(\times 10^{-10} \mathrm{erg} \mathrm{cm}^{-2} \mathrm{~s}^{-1}\right)\end{array}$ & $\begin{array}{l}\text { Phase } \\
\text { outburst }\end{array}$ \\
\hline 58352 & $1.79 \pm 0.03$ & $1.79 \pm 0.02$ & - & $<2$ & $1.13 \pm 0.05$ & $<0.1$ & $1.16 \pm 0.07$ & $0.830_{-0.009}^{+0.020}$ & $\mathrm{D}$ \\
\hline 58353 & $1.75 \pm 0.01$ & $1.68 \pm 0.01$ & - & $<2$ & $1.08 \pm 0.03$ & $<0.1$ & $1.16 \pm 0.04$ & $0.815 \pm 0.008$ & D \\
\hline 58354 & $1.799 \pm 0.009$ & $1.71 \pm 0.01$ & - & $<3$ & $1.06 \pm 0.04$ & $<0.1$ & $1.11 \pm 0.05$ & $0.779_{-0.004}^{+0.009}$ & D \\
\hline 58358 & $1.69 \pm 0.02$ & $1.29 \pm 0.01$ & - & $<2$ & $0.89 \pm 0.04$ & $<0.1$ & $0.95 \pm 0.05$ & $0.710_{-0.006}^{+0.009}$ & D \\
\hline 58359 & $1.76 \pm 0.04$ & $1.30 \pm 0.01$ & - & $<0.6$ & $0.83 \pm 0.05$ & $<0.1$ & $0.88 \pm 0.07$ & $0.64 \pm 0.01$ & D \\
\hline 58360 & $1.79 \pm 0.02$ & $1.33 \pm 0.01$ & - & $<0.5$ & $0.83 \pm 0.03$ & $<0.1$ & $0.89 \pm 0.04$ & $0.627 \pm 0.007$ & D \\
\hline 58361 & $1.79 \pm 0.02$ & $1.26 \pm 0.01$ & - & $<0.8$ & $0.78 \pm 0.03$ & $<0.02$ & $0.83 \pm 0.03$ & $0.582_{-0.002}^{+0.006}$ & D \\
\hline 58362 & $1.76 \pm 0.03$ & $1.24 \pm 0.01$ & - & $<0.7$ & $0.77 \pm 0.02$ & $<0.09$ & $0.82 \pm 0.03$ & $0.579_{-0.005}^{+0.010}$ & D \\
\hline 58363 & $1.80 \pm 0.02$ & $1.19 \pm 0.02$ & - & $<2$ & $0.74 \pm 0.02$ & $<0.08$ & $0.82 \pm 0.02$ & $0.54 \pm 0.01$ & D \\
\hline 58364 & $1.79 \pm 0.02$ & $1.17 \pm 0.01$ & - & $<1$ & $0.73 \pm 0.03$ & $<0.1$ & $0.79 \pm 0.04$ & $0.549_{-0.007}^{+0.010}$ & D \\
\hline 58368 & $1.76 \pm 0.03$ & $1.04 \pm 0.02$ & - & $<4$ & $0.68 \pm 0.03$ & $<0.07$ & $0.72 \pm 0.04$ & $0.500_{-0.004}^{+0.020}$ & D \\
\hline 58373 & $1.83 \pm 0.03$ & $0.841 \pm 0.009$ & - & $<0.4$ & $0.49 \pm 0.03$ & $<0.06$ & $0.53 \pm 0.04$ & $0.363_{-0.003}^{+0.008}$ & D \\
\hline 58374 & $1.86 \pm 0.04$ & $0.78 \pm 0.01$ & - & $<0.3$ & $0.46 \pm 0.02$ & $<0.08$ & $0.50 \pm 0.03$ & $0.345_{-0.010}^{+0.007}$ & D \\
\hline
\end{tabular}

